\title{
Genome-wide investigation of the heat shock transcription factor (Hsf) gene family in Tartary buckwheat (Fagopyrum tataricum)
}

Moyang Liu ${ }^{1,2+}$, Qin Huang ${ }^{1 \dagger}$, Wenjun Sun ${ }^{1}$, Zhaotang Ma', Li Huang ${ }^{1}$, Qi Wu ${ }^{1}$, Zizhong Tang ${ }^{1}$, Tongliang Bu', Chenglei $\mathrm{Li}^{1}$ and Hui Chen ${ }^{1 *}$ (i)

\begin{abstract}
Background: Heat shock transcription factor (Hsfs) is widely found in eukaryotes and prokaryotes. Hsfs can not only help organisms resist high temperature, but also participate in the regulation of plant growth and development (such as involved in the regulation of seed maturity and affects the root length of plants). The Hsf gene was first isolated from yeast and then gradually found in plants and sequenced, such as Arabidopsis thaliana, rice, maize. Tartary buckwheat is a rutin-rich crop, and its nutritional value and medicinal value are receiving more and more attention. However, there are few studies on the Hsf genes in Tartary buckwheat. With the whole genome sequence of Tartary buckwheat, we can effectively study the Hsf gene family in Tartary buckwheat.

Results: According to the study, $29 \mathrm{Hsf}$ genes of Tartary buckwheat (FtHsf) were identified and renamed according to location of FtHsf genes on chromosome after removing a redundant gene. Therefore, only 29 FtHsf genes truly had the functional characteristics of the FtHsf family. The 29 FtHsf genes were located on 8 chromosomes of Tartary buckwheat, and we found gene duplication events in the FtHsf gene family, which may promote the expansion of the FtHsf gene family. Then, the motif compositions and the evolutionary relationship of FtHsf proteins and the gene structures, cis-acting elements in the promoter, synteny analysis of FtHsf genes were discussed in detail. What's more, we found that the transcription levels of FtHsf in different tissues and fruit development stages were significantly different by quantitative real-time PCR (qRT-PCR), implied that FtHsf may differ in function.

Conclusions: In this study, only 29 Hsf genes were identified in Tartary buckwheat. Meanwhile, we also classified the FtHsf genes, and studied their structure, evolutionary relationship and the expression pattern. This series of studies has certain reference value for the study of the specific functional characteristics of Tartary buckwheat Hsf genes and to improve the yield and quality of Tartary buckwheat in the future.
\end{abstract}

Keywords: Tartary buckwheat, FtHsf genes, Genome-wide, Expression patterns, Evolution

\footnotetext{
* Correspondence: chenhui@sicau.edu.cn

${ }^{+}$Moyang Liu and Qin Huang contributed equally to this work.

'College of Life Science, Sichuan Agricultural University, Ya'an, China

Full list of author information is available at the end of the article
}

(c) The Author(s). 2019 Open Access This article is distributed under the terms of the Creative Commons Attribution 4.0 International License (http://creativecommons.org/licenses/by/4.0/), which permits unrestricted use, distribution, and reproduction in any medium, provided you give appropriate credit to the original author(s) and the source, provide a link to the Creative Commons license, and indicate if changes were made. The Creative Commons Public Domain Dedication waiver (http://creativecommons.org/publicdomain/zero/1.0/) applies to the data made available in this article, unless otherwise stated. 


\section{Background}

High temperature affects the growth, development and metabolism of plants [1-4]. Heat shock transcription factors are the main regulator of heat stress response, and it is important for eukaryotes and prokaryotes to resist high temperature [5-8]. When in a hot environment, Hsfs activate heat shock proteins (Hsps) by binding to the heat stress elements (HSEs) in Hsps promoter to resist high temperature [7, 9-14]. There is a ubiquitous heat shock response mechanism in plants, which includes a series of complex reactions, such as new protein synthesis, folding, specific biological functions and so on. In these proteins, Hsps as molecular chaperones, are essential to maintaining or restoring protein homeostasis [15-19].

A typical Hsf protein contains five domains, including a DNA-binding domain (DBD), an oligomerization domain (OD) or hydrophobic repeat domain (HR-A/ B) $[20,21]$, a nuclear localization signal domain (NLS), a nuclear export signal domain (NES) and an activator motif (AHA) [20, 22, 23]. Because of the differences in the HR-A/B domain of Hsf family members, the Hsf genes are divided into three big groups, named $\mathrm{A}$ (from A1 to A10), B (from B1 to B4) and C (from C1 to $\mathrm{C} 2$ ). It is worth noting that there is a AHA region which only exists in some members of group $\mathrm{A}$, and the AHA region is the key area for Hsfs to play a selfactivating role [21, 24].

Tartary buckwheat is a widely cultivated dicotyledonous nutritious food crop. Tartary buckwheat fruit contains abundant and balanced essential amino acids, and its total protein content is richer than that of main grain crops [25-28]. The Hsfs not only play a key role in plants resistance to high temperatures and improvements of plants heat tolerance, but also can regulate the growth and development of plants [29]. The Hsf genes family have been studied in many plants, and these studies were based on the heat stress response of Hsfs [22, 30, 31], but there were few studies on the regulation of plant growth and development by Hsfs. Because of the important role of Hsf genes in various phylogenetic and its resistance to high temperature (such as involved in the regulation of seed maturity and affects the root length of plants [5, 32]), it is of great significance to have a detailed study on the Tartary buckwheat Hsf gene family. Thanks to the complete genome sequencing of Tartary buckwheat, we can systematically research the $H s f$ gene family on the whole genome level. In this study, we firstly introduced the gene structures, cis-acting elements in the promoter, chromosomal locations, homology analysis, expression patterns of 29 Tartary buckwheat $H s f$ genes and motif compositions and phylogenetic analysis of 29 Tartary buckwheat Hsf proteins in detail. Secondly, the synteny analysis and phylogenetic relationships of $H s f$ genes between Fagopyrum tataricum and Beta vulgaris, Glycine max, Helianthus annuus, Oryza sativa, Solanum lycopersicum, Vitis vinifera, Arabidopsis thaliana were compared. Then, the expression patterns of the $H s f$ genes in different tissues were determined by qRT-PCR. More importantly, we also measured the transcriptional level of $H s f$ genes during fruit development. To sum up, this research provides valuable clues for studying the action mechanism of some members of the FtHsf gene family during buckwheat growth and development.

\section{Methods}

\section{Plant growth}

XIQIAO is one of buckwheat varieties, and it is rich in rutin. Since 2013, XIQIAO has grown under the same experimental conditions in the experimental base locate at the farm, Sichuan Agricultural University [33]. As for the experimental samples, we collected the materials including the fruits from three different stages $(13,19$, and 25 days after pollination, DAP), the flowers, the stems, the roots, and the leaves from five strains of Tartary buckwheat in the same physiological state [34]. The collected samples were stored in $-80^{\circ} \mathrm{C}$ refrigerator for subsequent study.

\section{Genes identification}

The genome sequence of Tartary buckwheat genome was obtained from the Tartary Buckwheat Genome Project. Firstly, the candidate Hsf proteins of Tartary buckwheat were authenticated by a BLASTp search. Then, we downloaded the Hsf domain (PF00447) from the Pfam database. According to the HMMER3, we used this date to build a HMM file. Finally, Hsf proteins were used as initial queries on the NCBI protein database (https://blast.ncbi.nlm.nih.gov/Blast.cgi? PROGRAM = blastp\&PAGE_TYPE = BlastSearch\&LINK LOC = blasthome) by BLASTp, further verifying that $\mathrm{Hsf}$ proteins derived from Tartary buckwheat belong to the Hsf gene family. The results showed that $29 H s f$ genes were identified as heat transcription factors of Tartary buckwheat. Besides, the isoelectric point, sequence length and molecular weight were acquired through the ExPasy (https://web. expasy.org/protparam/), and the subcellular localization of the Hsf proteins identified were obtained using CELLO (http://cello.life.nctu.edu.tw/) (Additional file 1).

\section{Phylogenetic analysis}

The Hsfs of Arabidopsis thaliana and the Hsfs of Tartary buckwheat were constructed into a phylogenetic tree by Neighbor-Joining (NJ) method, and all Hsfs were divided into three big groups. In addition, we constructed a multi-species phylogenetic evolutionary tree including FtHsf protein sequences and Vitis vinifera, Solanum lycopersicum, Oryza sativa, Arabidopsis thaliana, Beta vulgaris, Glycine max and Helianthus annuus Hsfs 
protein sequences that were downloaded from the UniProt database.

\section{Genetic structure, motifs composition and analysis of cis- acting elements}

By studying the conserved motifs in FtHsf protein, the structural differences among different FtHsf genes were found (Additional file 2). We compared several protein sequences, and the exon-intron structures of the FtHsf genes were understood by comparing the predicted coding sequence with the corresponding full-length sequence by the Gene Structure Display Server online program. Eventually, we have known ten conserved motifs of the recognized Hsf proteins according to the MEME online program. Additionally, PlantCARE software (http://bioinformatics.psb.ugent.be/webtools/plantcare/html/?tdsourcetag=s_pcqq_aiomsg) was used to predict the cis-acting elements of $2000 \mathrm{bp}$ upstream of all extended genes.

\section{Chromosomal distribution and gene duplication}

We used Circos to process the chromosomal location information of the FtHsf genes. We made use of Multiple collinear scanning toolkits (MCScanX) to detect the gene replication events. The homology analysis maps of Tartary buckwheat were drawn up by the Dual Synteny Plotter software. And the homology relationships between the homologous $H s f$ genes and other varieties of Tartary buckwheat were revealed [34].

\section{Gene expression analysis}

Firstly, the RNA of all samples was extracted with the EASYspin Plant RNAiso reagent (Aidlab, China). The cDNA was produced by $1 \mathrm{mg}$ RNA sample with a Prime Script RT Reagent Kit with gDNA Eraser (TaKaRa) with SYBR Premix Ex Taq II (TaKaRa). Expression pattern of FtHsf genes identified in different tissues (stems, roots, leave, fruits and flowers) and fruits at three different stages (13, 19 and 25 DAP) from five strains of Tartary buckwheat were analyzed with qRT-PCR, and each Tartary buckwheat was analyzed three times [35]. The qRTPCR primers of FtHsf genes listed in Additional file 4: Table S4 were obtained by Primer3 software (Additional file 4). We made the Tartary buckwheat $\mathrm{H} 3$ genes as the internal reference. The correlative expression data were calculated according to the $2^{-(\Delta \Delta \mathrm{Ct})}$ method [34].

\section{Subcellular localization}

In order to verify the above subcellular localization prediction, we selected two FtHsf genes (FtHsf18 and FtHsf19) as representatives to carry out subcellular localization experiments. First, the expression vectors of green fluorescent protein (GFP) tags were constructed [36], then the coding regions of FtHsf18 and FtHsf19 were amplified by PCR with specific primers and fused into the $\mathrm{N}$-terminal of GFP under the control of the CaMV35S promoter. Finally, the subcellular localization of the GFP expression in Arabidopsis protoplasts was observed with the help of confocal microscope after $12 \mathrm{~h}$ of transformation [37].

\section{Statistical analysis}

We processed and analyzed all the above data with the variance analysis with the Origin Pro 2018b statistics program and compared them by the least significant difference (LSD).

\section{Results \\ Identification of the FtHsf genes in Tartary buckwheat}

We used twice BLASTp methods to identify 29 FtHsf genes from the Tartary buckwheat genome after deleting redundant FtHsf genes because of the genome-wide shotgun strategy (Additional file 1). In this article, we renamed the FtHsf genes according to their chromosome locations, naming them from FtHsf1 to FtHsf29 (Additional file 1).

We provided the gene characteristics including CDS, Mw, pI and subcellular localization. The 29 predicted FtHsf proteins ranged from 216 amino acids (FtHsf5) to 503 amino acids (FtHsf17). The Mw of the Hsf proteins ranged from 24.59 (FtHsf5) to 55.30 (FtHsf17) $\mathrm{kDa}$, and the pI ranged from 4.77 (FtHsf5) to 9.1 (FtHsf6) (Additional file 1). The results subcellular localization showed that Hsf proteins were all situated in the nuclear (Additional file 1).

\section{Phylogenetic analysis and classification of the FtHsf genes} To investigate the phylogenetic relationship of the Tartary buckwheat Hsf proteins, we constructed a phylogenetic tree consisting of Arabidopsis thaliana (21 Hsf proteins) and Tartary buckwheat (29 Hsf proteins) (Fig. 1). According to the differences in the HR-A/B domain and phylogenetic relationships of FtHsf family members, the FtHsf genes were further divided into 3 big groups (named A, B and $C$ ) and 13 subfamilies, including A (A1, A2, A3, A4, A5, A6, A7, A8), B (B1, B2, B3, B4), and C1 (Figs. 1 and 2a). Tartary buckwheat is a dicotyledonous plant, and A9 and C2 only exist in monocotyledonous plants [22]. The B4 subfamily contained the largest number of FtHsf members, with five members. There were followed by A1, A4, A6 and A7 subgroups, all of which had three members of the FtHsf family. Then A2, B2, B3 and C1 subgroups all contained two members of the FtHsf family. Finally, A3, A5, A8 and B1 subgroups all contained only one member of the FtHsf family (Fig. 1). 


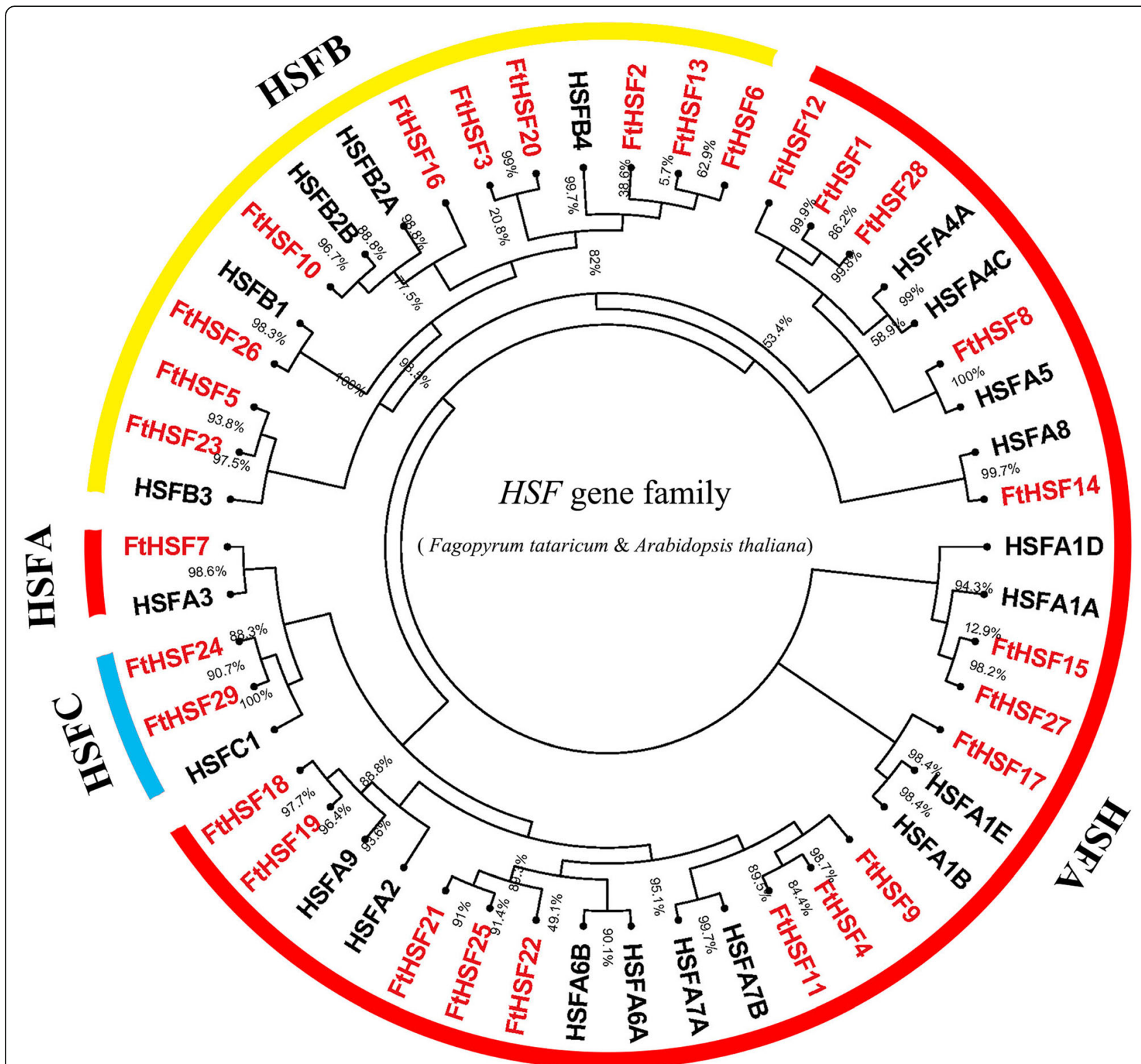

Fig. 1 Unrooted phylogenetic tree representing the relationships among the Hsf genes of Tartary buckwheat and Arabidopsis. As shown in the figure, the phylogenetic tree is divided into 3 groups, including group A, B and C

\section{Gene structure, motif composition and cis-acting} elements

In order to study the structural composition of FtHsf genes, we studied the exon and intron in detail including their amount and distribution (Fig. 2b). Gene structure analysis showed that the number of introns in different FtHsf genes was not the same. Most FtHsf genes only contained one intron, and four FtHsf genes (FtHsf2, FtHsf5, FtHsf6 and FtHsf9) contained two introns (Fig. 2b). The members of the same subfamily usually had similar exon / intron structures in terms of intron number and the exon length.
To further study the characteristic regions of the FtHsf proteins, the motifs of the Tartary buckwheat FtHsf proteins were analyzed by online MEME. According to the results of the MEME motif analysis, a schematic diagram was constructed to characterize the structures of the FtHsf proteins (Fig. 2c). According to the amino acid conserved sequences of the motifs $1,2,3,4,6,9$ and 10 , they were divided into five categories (DBD, HR-A/B or OD, NLS, NES and AHA) (Fig. 2c, Additional file 2) [31]. It can be seen from the Fig. $3 \mathrm{c}$ that group A FtHsf members had the most conserved motifs, followed by group $\mathrm{B}$ and group $\mathrm{C}$ FtHsf members. Motifs 1 and 2 (DBD domain) were both found in 27 members of the FtHsf family, but only motif 1 


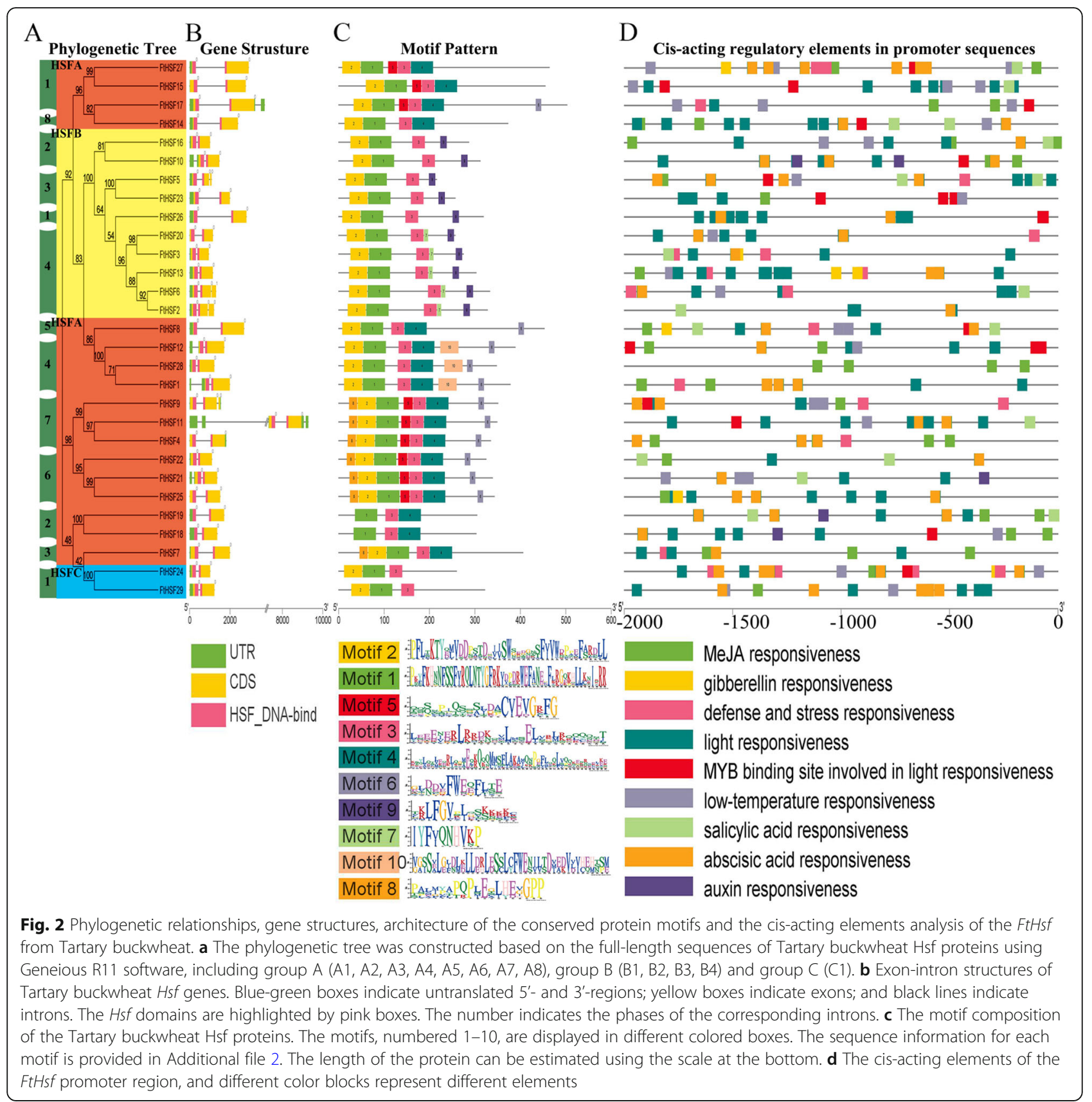

was found in FtHsf18 and FtHsf19. The DBDs included $4 \beta$ rotation angles and $3 \alpha$ helices in the $\mathrm{N}$-terminal region $(\alpha 1-\beta 1-\beta 2-\alpha 2-\alpha 3-\beta 3-\beta 4)$ (Fig. 3). And the helix motif (H2$\mathrm{T}-\mathrm{H} 3)$ can specifically bind to the promoter of heat stress inducible gene, but the length of the DBD domain varies greatly [22]. The conserved motifs 3 and 4 after DBD domain were HR-A/B region, which was found in all members of the FtHsf family. Specially, we found the length of class A FtHsfs were longer than that of class B and class C FtHsfs (Fig. 2c, Additional file 2). And the reason for this is that all class $\mathrm{A}$ and class $\mathrm{C}$ FtHsf members have an expanded HR-A/B region [31]. The NLS domain contained conserved motifs 3 and 9, it existed in all members FtHsf family. However, only motif 3 was used to represent NLS domain in class A and class C, while NLS domain was represented by both motifs 3 and 9 in class B. The conserved motif 10 belongs to the NES region, but it only appeared in three Class A members (FtHsf1, FtHsf12 and FtHsf28) (Fig. 2c, Additional file 2). Therefore, all of 29 FtHsfs have NLS domain, but only three Class A members contain NES domain, and the two domains jointly maintain the balance of FtHsf inside and outside the nucleus $[23,31]$. The conserved motif 6 was identified as a characteristic AHA domain, which is a structure that is unique to the group A 


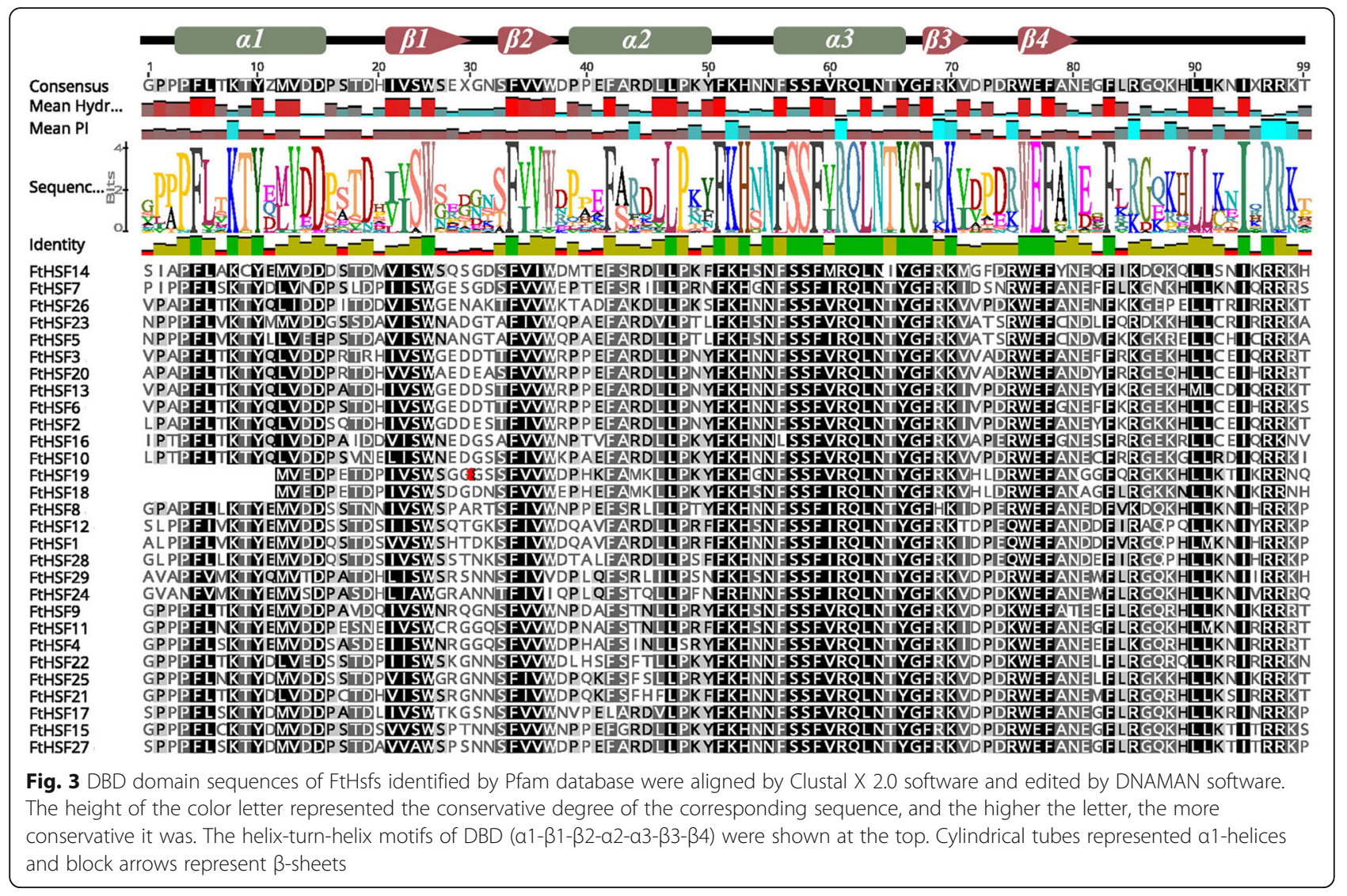

family, while no AHA domain was found in group B or in group C (Fig. 2c, Additional file 2). Additionally, there are other conserved motifs in FtHsfs, but the action mechanism of these motifs is unclear. All in all, the conserved motif composition and the gene structure within the same group of FtHsf members were very similar, and the results of phylogenetic analysis supported the reliability of the population classification (Fig. 2, Additional file 2).

By analyzing the cis-acting elements in the promoter region, we found that most FtHsf genes contained multiple Light-responsive elements, ABA-responsive elements and MeJA-responsive elements. Nearly 50\% of FtHsf genes contained Low-temperature responsive element, MYB-responsive element, Salicylic acidresponsive element and Defense and Stress responsive element, while only about $20 \%$ of FtHsf genes contained Auxin-responsive element and Gibberellinresponsive element (Fig. 2d). It can be inferred that FtHsf can not only participate in a variety of abiotic stress responses [38, 39], but also respond to a variety of exogenous hormones [40].

Chromosomal distribution and homology analysis According to the study, there are eight chromosomes in Tartary buckwheat, and each chromosome has a different number of the FtHsf genes (Fig. 4). FtHsf genes were found in all chromosomes, among which the most FtHsf genes were found on chromosome 3 and chromosome 4, but chromosome 2 and chromosome 5 had only two FtHsf genes (Fig. 4). According to Holub, a chromosome region containing more than two genes within $200 \mathrm{~kb}$ is defined as a tandem duplication [41]. Homology analysis showed that there were no tandem duplication event sequences in the Tartary buckwheat (Fig. 5). Of the 29 FtHsf genes, 13 pairs of fragment duplication were found, with the most duplication events on chromosome 1 and chromosome 6 and only one on chromosome 4 and chromosome 5 (Fig. 5). These results showed that gene duplication may be the cause of the formation of some FtHsf genes and that these fragment duplication events were the main cause of $F t H s f s$ evolution [42].

Evolutionary and synteny analysis of the FtHsfs and the Hsfs of several different species

To further study the evolutionary relationship between the FtHsf genes, we used MEGA 5.0 to construct a phylogenetic tree that consisted of 8 representative species of Hsf protein sequences, including one monocotyledonous (Oryza sativa) and seven dicotyledonous plants (Vitis vinifera, Solanum lycopersicum, Arabidops is thaliana, Beta vulgaris, Glycine max, Helianthus annuus and Fagopyrum tataricum) (Fig. 6). According to the phylogenetic tree, 


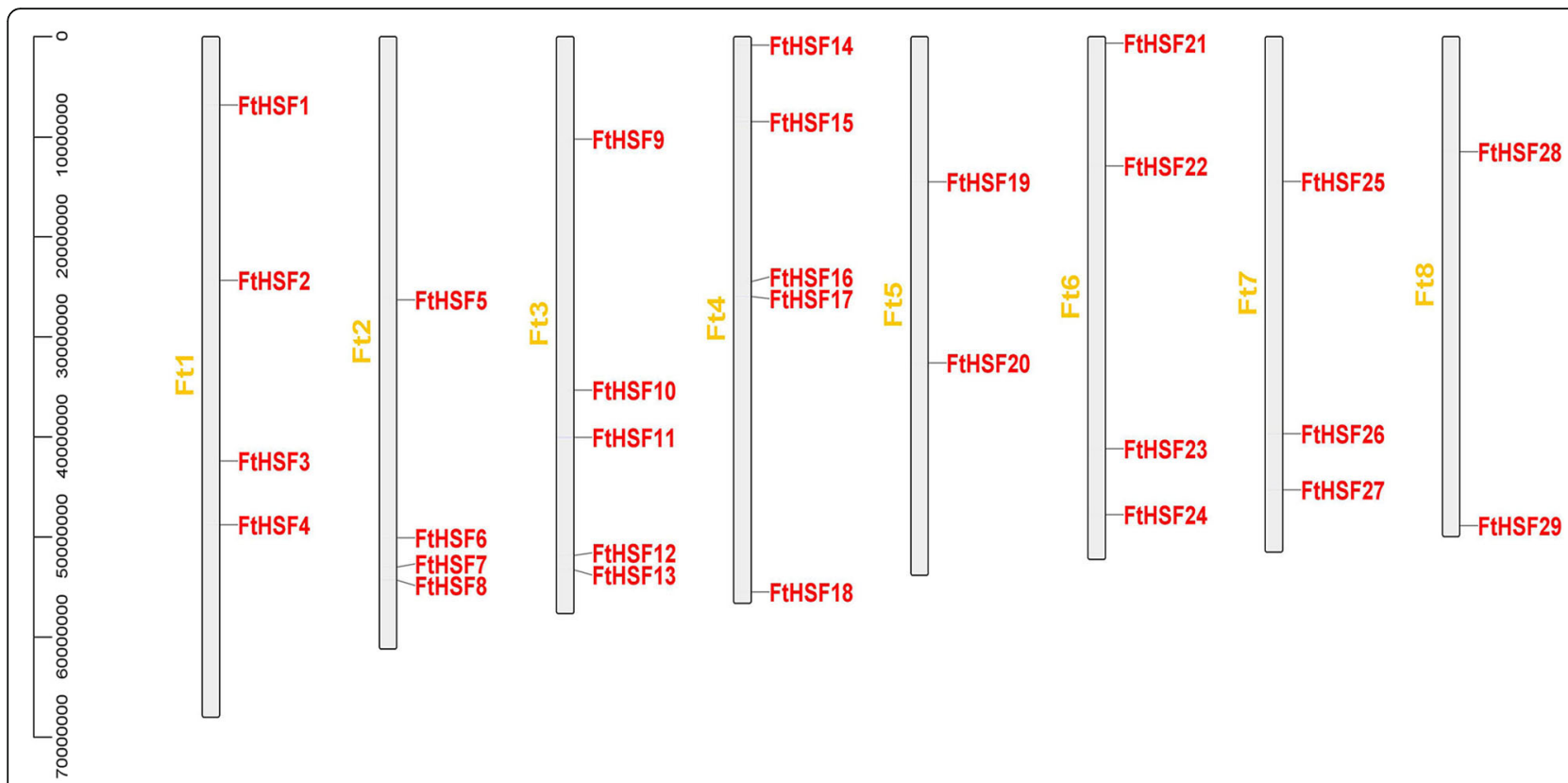

Fig. 4 Schematic representations of the chromosomal distribution of the Tartary buckwheat Hsf genes. The number of the chromosome is shown on each chromosome

Hsf members of the same subclass from different species gather together, and the Hsfs were divided into three big groups, named A, B and C (Fig. 6). Using MEME web servers, we searched the conserved motifs shared by the Hsf proteins. Finally, we obtained ten different conserved motifs and classified them according to their conservative sequence (Fig. 6, Additional file 2) [31]. Among which motif 1 , motif 2 , motif 4 and motif 6 encoded the $D B D$ domain, motif 5 and motif 3 belonged to HR-A/B, and the motif 7 represented the AHA domain (Fig. 6, Additional file 2). Almost all Hsf families have motif 1, 2, 4 and 6, motif 3 and 5, indicating that DBD domain and HR-A/B domain were very conservative in $\mathrm{Hsf}$ families (Fig. 6). Motif 7 only existed in some members of Class A Hsf family (Fig. 6), the AHA region was the key area for Hsfs to play a self-activating role, and it was speculated that the mechanism of Hsfs self-activation was similar in different plants [21, 23]. As shown in Fig. 5, the Hsfs of the same subclass in different species usually had the same motifs composition (such as FtHsf3 and Solyc11g064990.1.1), it was speculated that there may be similar functions between proteins.

To understand more about the phylogeny of Tartary buckwheat FtHsf genes family, the Hsf gene of the Tartary buckwheat was subjected to a synteny analysis with the $H s f$ gene of the other seven typical plants, including six dicotyledonous plants (Arabidopsis thaliana, Beta vulgaris, Glycine max, Helianthus annuus, Solanum lycopersicum, and Vitis vinifera) and a monocotyledonous plant (Oryza sativa) (Fig. 7). There were 23 FtHsf genes that were synchronized with those in Glycine max, and then there was Solanum lycopersicum (20), Vitis vinifera (18), Beta vulgaris (13), Arabidopsis thaliana (11), Helianthus annuus (7), and Oryza sativa (7) (Fig. 7, Additional file 3). The number of homologous pairings of the other 6 species (Glycine max, Solanum lycopersicum, Vitis vinifera, Oryza sativa, Arabidopsis thaliana, Beta vulgaris and Helianthus annuus) were 67, 31, 20, 19, 16, 14, and 8 (Fig. 7, Additional file 3). The results showed that the genetic relationship between Tartary buckwheat $H s f$ genes and soybean $H s f$ genes was close. At the same time, we could find that some FtHsf genes were associated with multiple $H s f$ genes in other species, for example, the FtHsf11 of buckwheat was associated with five $H s f$ genes in soybean and the rice, respectively (Fig. 7, Additional file 3). The FtHsf11 may play a significant role in the evolution of the $F t H s f$ gene family.

\section{Expression patterns of FtHsf genes in different plant tissues}

The qRT-PCR was used to determine the expression of 29 FtHsf genes in different tissues and the physiological functions of FtHsf genes were discussed. (Fig. 8). The results showed that there were significant differences in the expression of the FtHsf genes in different tissues/organ, showing that the FtHsfs had a variety of functions in the growth and development of Tartary buckwheat. Some FtHsf genes had prominent expression in Tartary Buckwheat tissues/organ. Three FtHsf genes (FtHsf18/FtHsf19/FtHsf22) were highly expressed in fruit (Fig. 8). Seven FtHsf genes (FtHsf10/ FtHsf9/FtHsf6/FtHsf15/FtHsf4/FtHsf16/FtHsf5) were high expression in the flowers than in the other 


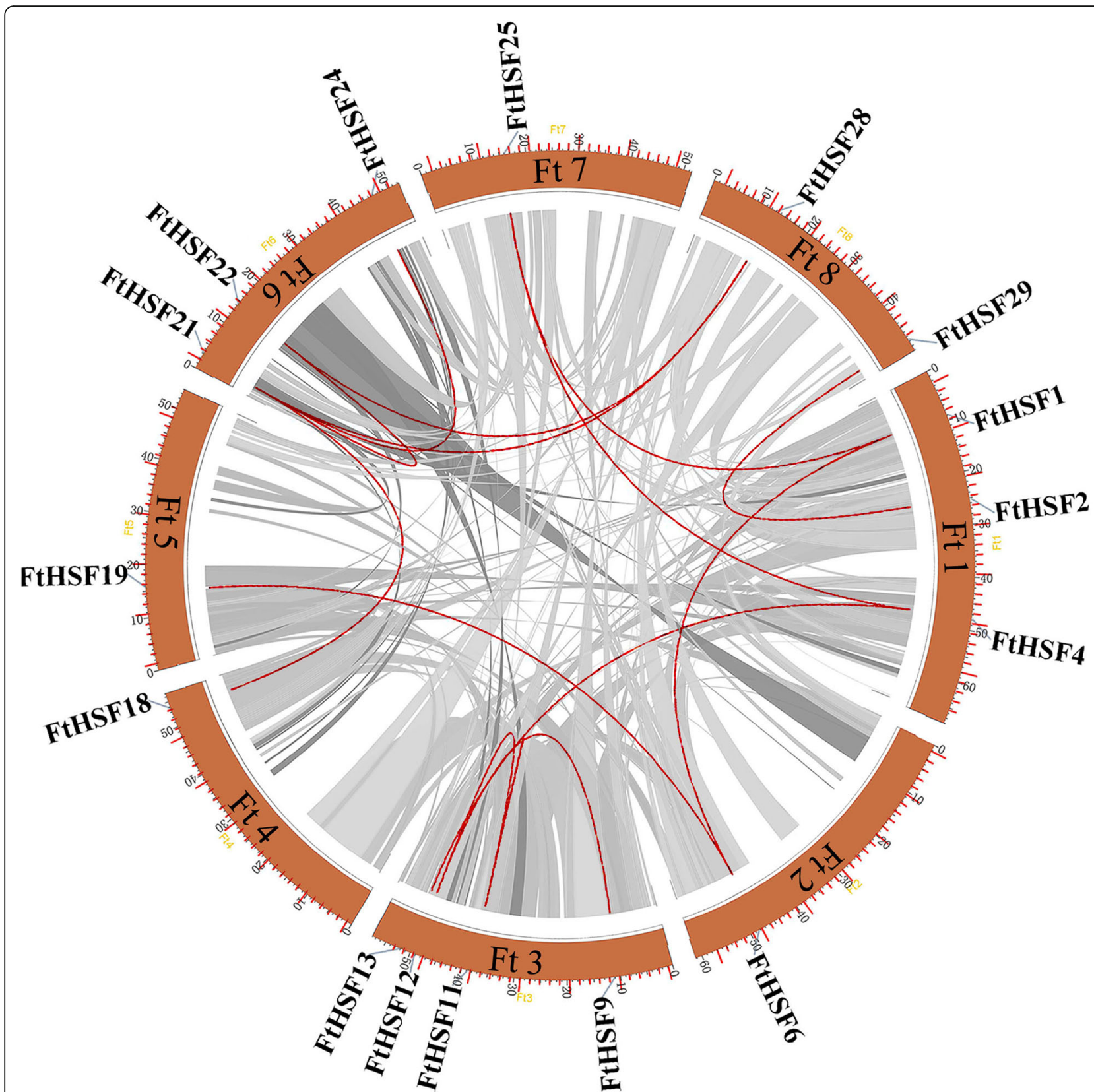

Fig. 5 Schematic diagram of the relationship between different FtHsf genes on different chromosomes. Grey and red lines indicate all homology blocks in the Tartary buckwheat genome, and the red lines indicate duplicated FtHsf gene pairs

tissues/organs. According to the study, we could find that many FtHsf genes were highly expressed in the leaves (except FtHsf20/FtHsf5) (Fig. 8). The majority of the FtHsf genes were expressed in Tartary buckwheat stems except FtHsf3. Besides, we also studied the correlations among the FtHsf genes expression patterns in the roots, stems, flowers, leaves and fruit of Tartary buckwheat (Fig. 9). The results showed that many FtHsf genes belonged to the positive correlation, and it was worth noting that there was a significant positive correlation between some FtHsf genes, for example, FtHsf18 and FtHsf19, FtHsf12 and FtHsf29, FtHsf5 and FtHsf9 and so on (Fig. 10).

\section{Differential expression of the FtHsf genes during fruit development in Tartary buckwheat}

To explore the possible role of the FtHsf genes in different stages of Tartary buckwheat fruit development, we compared the gene expression patterns at different buckwheat fruit development stages $(13,19$, and 25 days after pollination, DAP) (Fig. 10) [25]. As the results show, all members of the FtHsf family were expressed at 


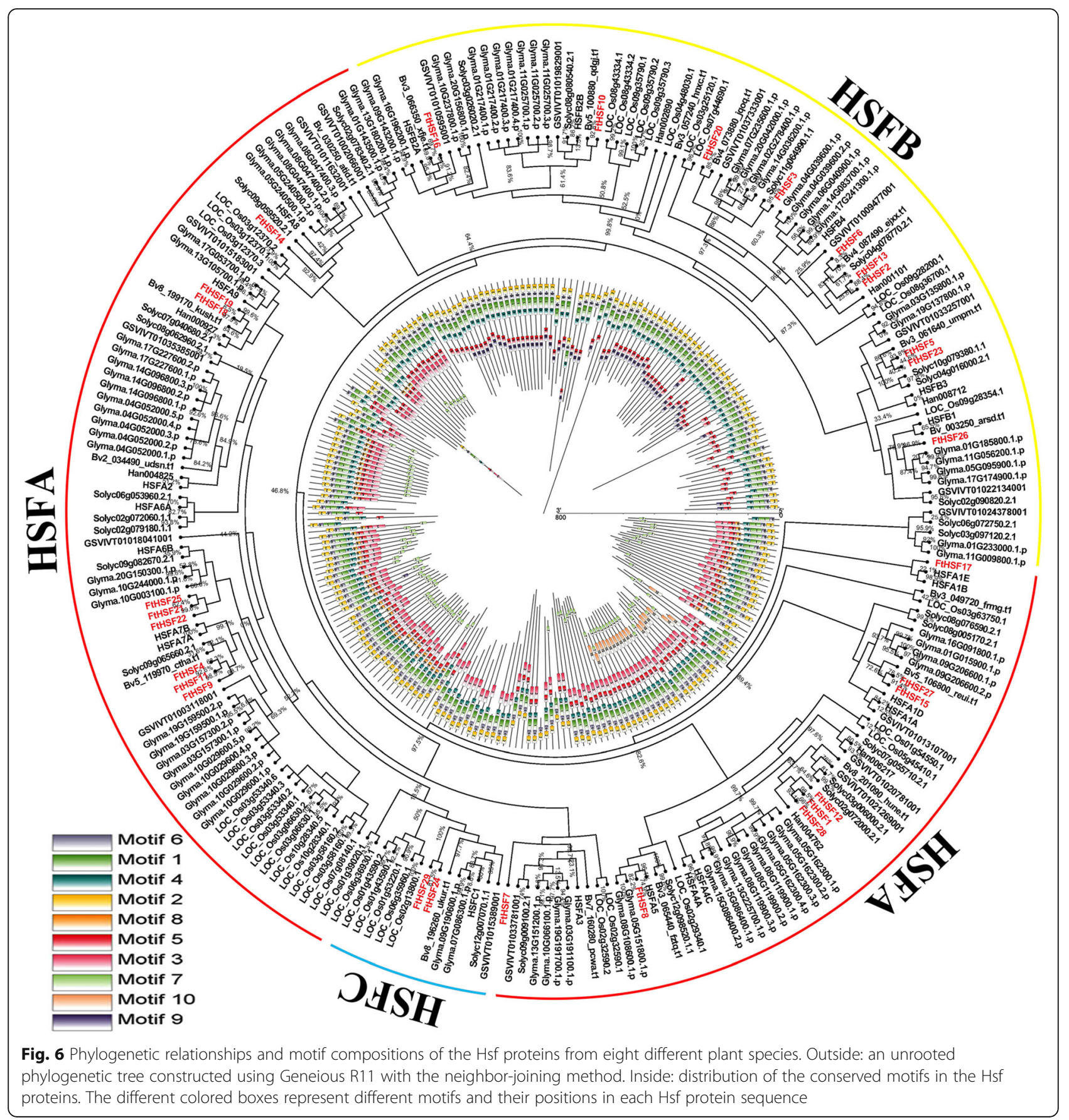

the whole stage of buckwheat fruit development, and the expression of most of the FtHsf genes has changed in these three stages (Fig. 10). The expression of some $F t H s f s$ showed obvious trend in different developmental stages. With the maturity of the Tartary buckwheat fruit, the expressions of $14 \mathrm{FtHsfs}$ were fluctuating up and down, the expression level of 11 FtHsfs decreased, and only 4 FtHsfs (FtHsf18, FtHsf19, FtHsf21 and FtHsf22) expression were on the rise (Fig. 10). It was speculated that these four FtHsf genes play a certain role in the ripening process of Tartary buckwheat fruits. At the same time, by studying the correlation between FtHsf genes and fruit development, and the correlation between $F t H s f$ genes in the process of fruit development (Fig. 11), we found that the expression of most genes was negatively correlated with fruit development, and only the FtHsf 19 gene showed significant positively correlated. At the same time, we found that most FtHsf genes were positively correlated with each other during fruit development of Tartary buckwheat, and there was a 

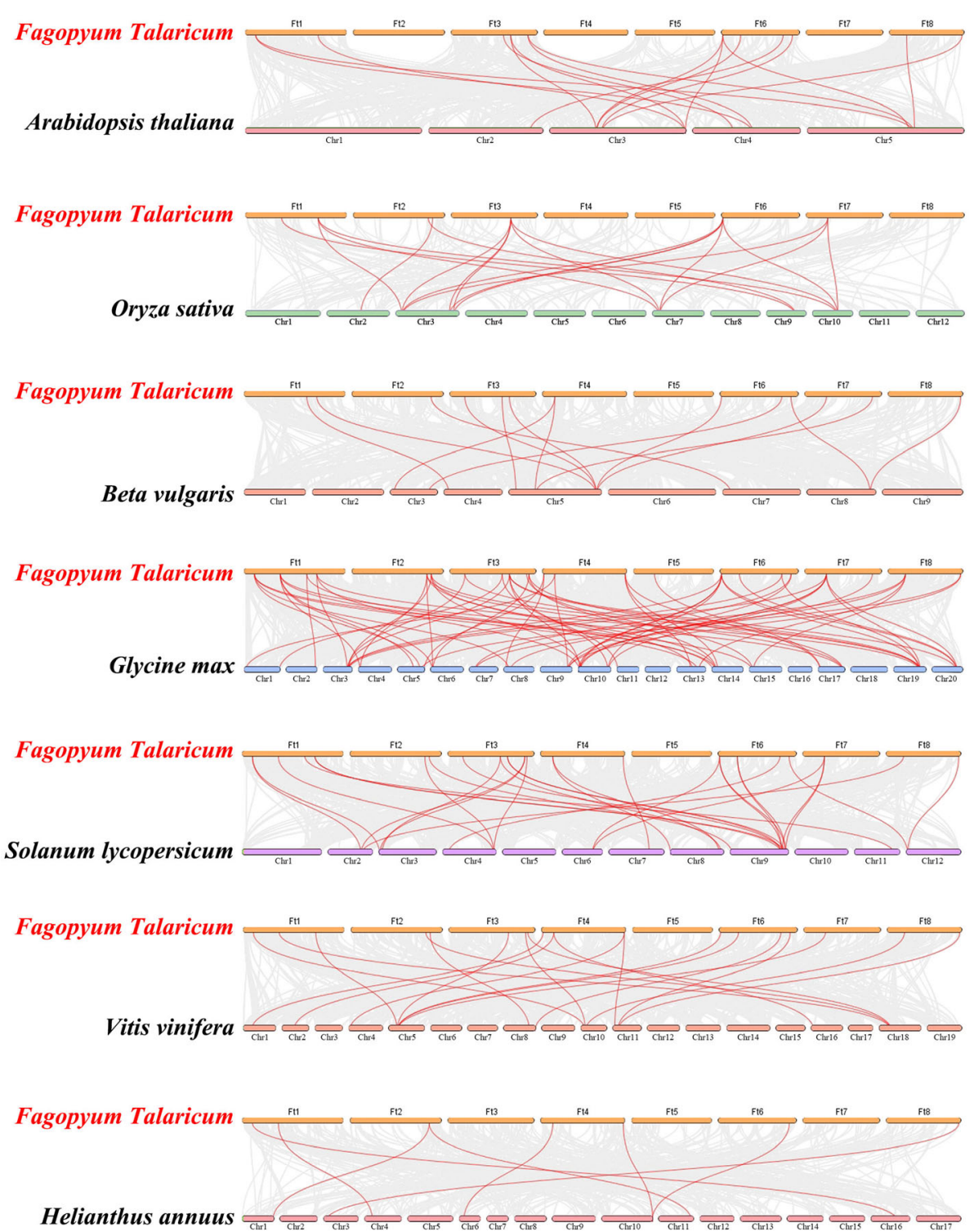

Fig. 7 Synteny analysis between the Hsf genes of Tartary buckwheat and seven representative plant species. Gray lines in the background indicate the collinear blocks within Tartary buckwheat and other plant genomes, while the red lines highlight the syntenic Hsf gene pairs

significant positive correlation between some of these genes (FtHsf18 and FtHsf21, FtHsf3 and FtHsf7, FtHsf1 and FtHsf14 and so on) (Fig. 11).

\section{FtHsf18 and FtHsf19 were localized in the nucleus}

In order to verify the prediction of subcellular localization, two FtHsf genes (FtHsf18 and FtHsf19) were selected as representatives to carry out the experiment (Fig. 12). Subcellular localization analysis was carried out by constructing the expression vector labeled with green fluorescent protein (GFP) [36], the expression of GFP in protoplasts vector of mesophyte cells of Arabidopsis thaliana was used as the control group, and the subcellular localization of GFP expression was observed by confocal microscope
[37]. It can be seen from Fig. 12 that the distribution of GFP in protoplasts of the control group was uniform, while the FtHsf18 and FtHsf19 fusion proteins were only located in the nucleus. This showed that FtHsf18 and FtHsf19 proteins were located in the nucleus, and the prediction of subcellular localization was correct.

\section{Discussion}

FtHsf genes identification and evolutionary analysis in Tartary buckwheat

$H s f$ genes are the heat stress transcription factors [31]. The number and motif composition of $H s f$ genes are often different in different species. It was reported that 28, 21, 19, 18,16 and $13 \mathrm{Hsf}$ genes were found in poplar 


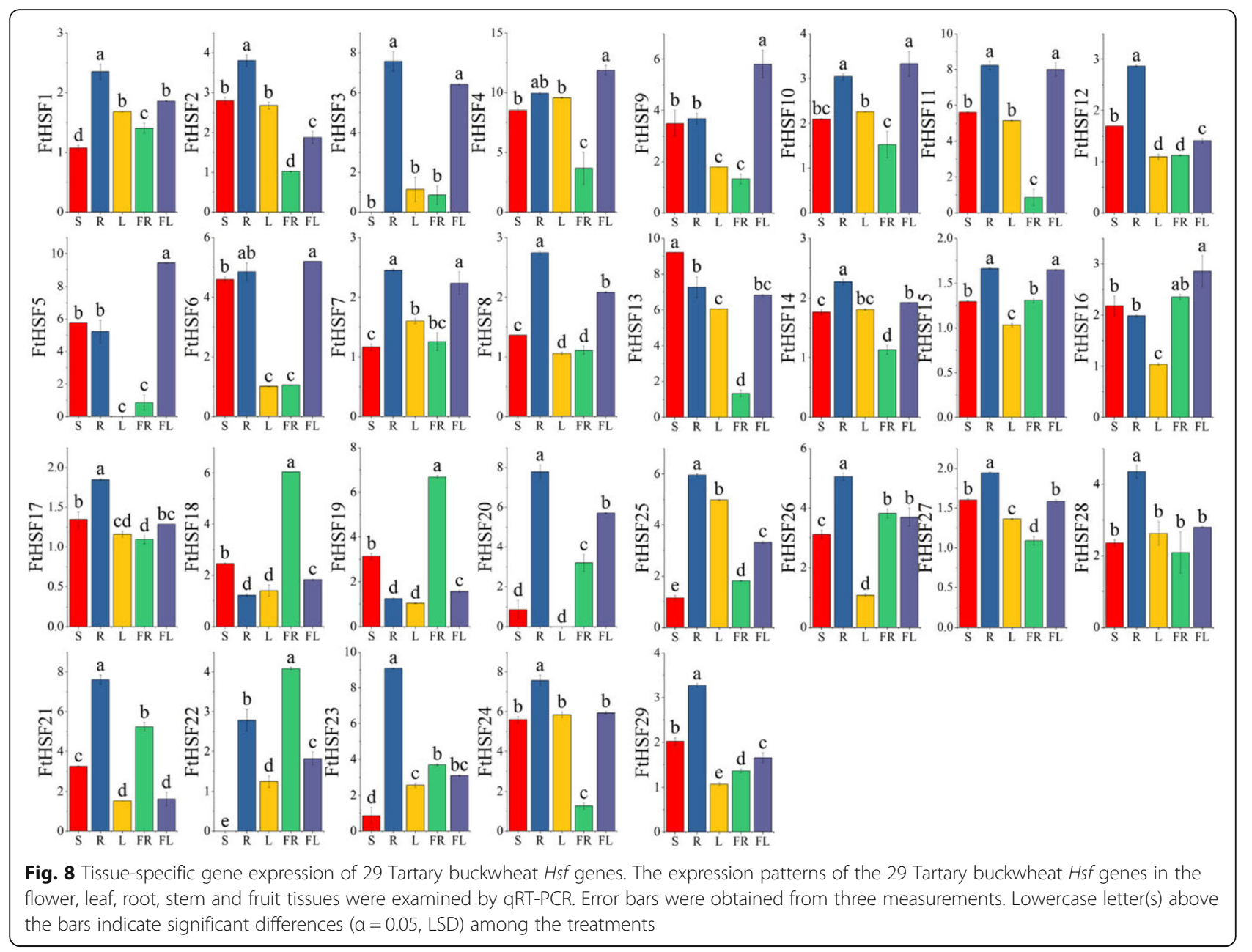

[24], Arabidopsis [21], grapes [43], tomatoes [31], alfalfa [24] and beets [44], respectively. In this research, we found $29 H s f$ genes in the Tartary buckwheat, and all the FtHsf proteins were located in the nucleus (Additional file 1, Fig. 12). Compared with other dicotyledonous plants, more $H s f$ genes were found in Tartary buckwheat genome. The recombination and expansion of the genome can usually alter the number of members of a gene family [25]. The genomic replication events which lead to the duplication of the gene family often occur during the evolution of angiosperms [25]. It was presumed that there were more genomic replication events in Tartary buckwheat after differentiation from the early ancestors of other species.

Different gene duplication patterns contribute to the amplification of corresponding gene families in plant genomes, such as genome duplication, tandem duplication and fragment duplication [45]. It is reported that some large gene families (such as WRKY families) are more likely to be amplified by fragment duplication and tandem duplication than by other replication events [46]. However, gene families such as MADS and NBS expand primarily through transposed duplications. Gene replication causes the emergence of more than 90\% regulatory genes in Arabidopsis thaliana [47]. In this article, the synteny analysis confirmed that the expansion of the FtHsf gene family in Tartary buckwheat mainly originated from fragment duplication, not from tandem duplication (Fig. 5) $[48,49]$. FtHsf21 and FtHsf22 belong to a pair of gene pairs duplications by fragments (Fig. 5). By studying their expression patterns, it was found that FtHsf21 was highly expressed in the roots, but FtHsf22 was highly expressed in the fruits (Fig. 8). Therefore, we could know that the specific expression of duplication genes was different. However, through the comparison of their motifs, it was found that their motifs were the same (Fig. 2). It was speculated that the reason for the differential expression of these genes may be due to a gene mutation during gene duplication, which resulted in the loss of function of some parts of the gene [50] (Additional file 2).

Fagopyrum tataricum, Arabidopsis thaliana, Beta vulgaris, Glycine max, Helianthus annuus, Solanum lycopersicum, and Vitis vinifera are dicotyledonous plants, and Oryza sativa is a monocotyledon plant. Phylogenetic tree 


\begin{tabular}{|c|c|c|c|c|c|c|c|c|c|c|c|c|c|c|c|c|c|c|c|c|c|c|c|c|c|c|c|c|c|c|}
\hline 1.0 & 0.49 & 0.90 & 0.49 & 0.21 & 0.29 & 0.94 & 0.81 & 0.33 & 0.73 & 0.61 & 0.63 & 0.06 & 0.69 & 0.60 & -0.05 & \begin{tabular}{|l|}
0.69 \\
\end{tabular} & -0.50 & -0.56 & 0.79 & \begin{tabular}{|l|}
0.42 \\
\end{tabular} & 0.38 & 0.88 & 0.57 & 0.90 & 0.43 & 0.60 & 0.88 & 0.57 & FtHSF1 & \\
\hline 0.4 & 1.00 & 0.40 & 0.56 & 0.17 & 0.47 & 0.47 & 0.58 & 0.22 & 0.48 & 0.71 & 0.80 & 0.74 & \begin{tabular}{|l|l}
0.88 \\
\end{tabular} & 0.20 & -0.41 & 0.83 & -0.80 & -0.73 & 0.24 & \begin{tabular}{|l|}
0.34 \\
\end{tabular} & -0.41 & 0.50 & \begin{tabular}{|l|l}
0.88 \\
\end{tabular} & 0.63 & \begin{tabular}{|l|}
0.14 \\
\end{tabular} & 0.86 & 0.80 & 0.73 & FtHSF2 & \\
\hline 0.9 & 0.40 & 1.00 & 0.61 & 0.60 & 0.63 & 0.98 & 0.94 & 0.67 & 0.89 & 0.75 & 0.66 & 0.21 & \begin{tabular}{|l|}
0.71 \\
\end{tabular} & 0.87 & 0.35 & 0.71 & $-0.49 \mid$ & -0.52 & 0.92 & 0.35 & 0.27 & 0.76 & 0.58 & 0.65 & 0.63 & 0.71 & 0.82 & 0.65 & FtHSF3 & \\
\hline 0.4 & 0.56 & 0.61 & 1.00 & 0.65 & 0.65 & 0.69 & 0.56 & 0.78 & 0.90 & 0.94 & 0.32 & 0.76 & 0.85 & 0.39 & 0.01 & 0.42 & $-0.92 \mid$ & -0.93 & 0.28 & -0.36 & $-0.55 \mid$ & 0.11 & 0.88 & 0.51 & -0.06 & 0.70 & 0.50 & 0.27 & FtHSF4 & \\
\hline 0.2 & 0.17 & 0.60 & 0.65 & 1.00 & 0.94 & 0.52 & 0.65 & 0.98 & 0.76 & 0.72 & 0.37 & 0.57 & 0.51 & 0.79 & 0.74 & 0.40 & -0.40 & -0.36 & 0.55 & -0.09 & -0.30 & 0.06 & 0.48 & $-0.08 \mid$ & 0.50 & 0.62 & 0.29 & 0.42 & FtHSF5 & \\
\hline 0.2 & 0.47 & 0.63 & 0.65 & 0.94 & 1.00 & 0.55 & 0.76 & 0.90 & 0.75 & 0.81 & 0.64 & 0.73 & 0.68 & 0.79 & 0.57 & 0.66 & -0.52 & -0.45 & 0.58 & 0.14 & -0.36 & 0.23 & 0.65 & 0.05 & 0.58 & 0.83 & 0.51 & 0.68 & FtHSF6 & \\
\hline 0.9 & 0.47 & 0.98 & 0.69 & 0.52 & 0.55 & 1.00 & 0.89 & 0.62 & 0.91 & 0.79 & 0.62 & 0.25 & 0.77 & 0.76 & 0.18 & 0.69 & $-0.60 \mid$ & -0.65 & 0.83 & 0.26 & 0.20 & 0.74 & 0.67 & 0.77 & 0.48 & 0.71 & 0.84 & 0.58 & FtHSF7 & \\
\hline 0.8 & 0.58 & 0.94 & 0.56 & 0.65 & 0.76 & 0.89 & 1.00 & 0.66 & 0.82 & 0.78 & 0.86 & 0.39 & 0.78 & 0.90 & 0.35 & 0.89 & -0.51 & -0.49 & 0.91 & 0.53 & 0.15 & 0.79 & 0.66 & 0.56 & 0.75 & 0.87 & 0.90 & 0.86 & FtHSF8 & \\
\hline 0.3 & 0.22 & 0.67 & 0.78 & 0.98 & 0.90 & 0.62 & 0.66 & 1.00 & 0.86 & 0.81 & 0.34 & 0.60 & 0.60 & 0.76 & 0.62 & 0.39 & $-0.53 \mid$ & -0.52 & 0.54 & -0.19 & -0.32 & 0.08 & 0.58 & 0.08 & 0.38 & 0.64 & $\mid 0.34$ & 0.37 & FtHSF9 & \\
\hline 0.7 & 0.48 & 0.89 & 0.90 & 0.76 & 0.75 & 0.91 & 0.82 & 0.86 & 1.00 & 0.93 & 0.51 & 0.54 & \begin{tabular}{|l|l}
0.84 \\
\end{tabular} & 0.73 & 0.27 & 0.59 & -0.76 & -0.78 & 0.67 & -0.05 & $|-0.17|$ & 0.43 & 0.79 & 0.57 & 0.33 & \begin{tabular}{|l|}
0.76 \\
\end{tabular} & 0.68 & 0.48 & FtHSF10 & \\
\hline 0.6 & 0.71 & 0.75 & 0.94 & 0.72 & 0.81 & 0.79 & 0.78 & 0.81 & 0.93 & 1.00 & 0.62 & $\mid 0.79$ & 0.95 & 0.59 & 0.10 & 0.70 & $-0.90 \mid$ & -0.88 & 0.50 & -0.03 & -0.43 & 0.36 & 0.94 & 0.55 & 0.24 & $0.90 \mid$ & 0.71 & 0.58 & FtHSF11 & 1.00 \\
\hline 0.6 & 0.80 & 0.66 & 0.32 & 0.37 & 0.64 & 0.62 & 0.86 & 0.34 & 0.51 & 0.62 & 1.00 & 0.46 & 0.74 & 0.65 & 0.07 & 0.99 & -0.45 & -0.38 & 0.70 & \begin{tabular}{|l|}
0.75 \\
\end{tabular} & 0.05 & 0.80 & 0.65 & 0.50 & 0.71 & 0.89 & 0.91 & 0.99 & FtHSF12 & 0.80 \\
\hline 0.0 & 0.74 & 0.21 & 0.76 & 0.57 & 0.73 & 0.25 & 0.39 & 0.60 & 0.54 & 0.79 & 0.46 & 1.00 & 0.77 & 0.20 & -0.06 & 0.51 & -0.82 & \begin{tabular}{|l|}
-0.73 \\
\end{tabular} & 0.00 & -0.18 & -0.85 & -0.08 & 0.84 & 0.14 & -0.04 & \begin{tabular}{|l|}
0.77 \\
\end{tabular} & 0.37 & 0.44 & FtHSF13 & 0.60 \\
\hline 0.6 & 0.88 & 0.71 & 0.85 & 0.51 & 0.68 & 0.77 & 0.78 & 0.60 & 0.84 & 0.95 & 0.74 & 0.77 & 1.00 & 0.49 & -0.14 & 0.81 & $-0.93 \mid$ & -0.90 & 0.47 & 0.14 & -0.38 & 0.51 & 0.98 & 0.70 & 0.22 & 0.94 & 0.84 & 0.68 & FtHSF14 & 0.40 \\
\hline 0.6 & 0.20 & 0.87 & 0.39 & 0.79 & 0.79 & 0.76 & 0.90 & \begin{tabular}{|l|l|} 
& 0.76 \\
\end{tabular} & 0.73 & 0.59 & 0.65 & 0.20 & 0.49 & 1.00 & 0.72 & 0.66 & $-0.20 \mid$ & -0.19 & \begin{tabular}{|c|}
0.94 \\
\end{tabular} & 0.44 & 0.27 & 0.60 & 0.36 & 0.21 & 0.87 & \begin{tabular}{|c|}
0.64 \\
\end{tabular} & 0.62 & 0.70 & FtHSF15 & 0.20 \\
\hline-0.0 & -0.41 & 0.35 & 0.01 & 0.74 & 0.57 & 0.18 & 0.35 & \begin{tabular}{|l|}
0.62 \\
\end{tabular} & 0.27 & 0.10 & 0.07 & -0.06 & -0.14 & 0.72 & 1.00 & 0.04 & 0.32 & 0.34 & 0.53 & 0.09 & 0.23 & -0.01 & $-0.22 \mid$ & $-0.48 \mid$ & 0.66 & \begin{tabular}{|c|}
0.08 \\
\end{tabular} & -0.09 & 0.18 & FtHSF16 & -0.00 \\
\hline 0.6 & 0.83 & 0.71 & 0.42 & 0.40 & 0.66 & 0.69 & 0.89 & 0.39 & 0.59 & 0.70 & 0.99 & 0.51 & \begin{tabular}{|l|l|}
0.81 \\
\end{tabular} & 0.66 & 0.04 & 1.00 & $-0.55 \mid$ & -0.49 & 0.71 & 0.68 & 0.01 & 0.80 & 0.72 & 0.58 & 0.66 & 0.92 & 0.94 & $0.97 \mid$ & FtHSF17 & -020 \\
\hline-0.5 & -0.80 & -0.49 & -0.92 & -0.40 & -0.52 & -0.60 & -0.51 & -0.53 & -0.76 & $\mid-0.90$ & -0.45 & $-0.82 \mid$ & $|-0.93|$ & $-0.20 \mid$ & 0.32 & -0.55 & 1.00 & 0.99 & -0.16 & 0.20 & 0.60 & -0.21 & $|-0.97|$ & $-0.65 \mid$ & 0.15 & -0.77 & -0.61 & -0.38 & FtHSF18 & -0 \\
\hline-0.5 & -0.73 & -0.52 & -0.93 & -0.36 & -0.45 & -0.65 & -0.49 & -0.52 & $\mid-0.78$ & $|-0.88|$ & -0.38 & -0.73 & $-0.90 \mid$ & -0.19 & 0.34 & $|-0.49|$ & 0.99 & 1.00 & $-0.17 \mid$ & 0.25 & 0.53 & -0.23 & $|-0.94|$ & $|-0.71|$ & 0.19 & $|-0.70|$ & $-0.60 \mid$ & $-0.30 \mid$ & FtHSF19 & $\cap 00$ \\
\hline 0.7 & 0.24 & 0.92 & 0.28 & 0.55 & 0.58 & 0.83 & 0.91 & 0.54 & 0.67 & 0.50 & 0.70 & 0.00 & 0.47 & 0.94 & 0.53 & 0.71 & -0.16 & -0.17 & 1.00 & 0.61 & 0.51 & 0.82 & 0.32 & 0.44 & 0.87 & 0.59 & 0.75 & 0.72 & FtHSF20 & \\
\hline 0.4 & 0.34 & 0.35 & -0.36 & -0.09 & 0.14 & 0.26 & 0.53 & -0.19 & -0.05 & -0.03 & 0.75 & -0.18 & 0.14 & 0.44 & 0.09 & 0.68 & 0.20 & 0.25 & 0.61 & 1.00 & 0.57 & 0.81 & 0.00 & 0.25 & 0.78 & 0.36 & 0.61 & 0.77 & FtHSF21 & \\
\hline 0.3 & -0.41 & 0.27 & -0.55 & -0.30 & -0.36 & 0.20 & 0.15 & -0.32 & $-0.17 \mid$ & -0.43 & 0.05 & $-0.85 \mid \cos$ & -0.38 & 0.27 & 0.23 & 0.01 & 0.60 & 0.53 & 0.51 & 0.57 & 1.00 & 0.57 & $-0.53 \mid$ & 0.16 & 0.49 & $|-0.31|$ & 0.14 & 0.07 & FtHSF22 & \\
\hline 0.8 & 0.50 & 0.76 & 0.11 & 0.06 & 0.23 & 0.74 & 0.79 & 0.08 & 0.43 & 0.36 & 0.80 & -0.08 & 0.51 & 0.60 & -0.01 & 0.80 & $-0.21 \mid$ & -0.23 & 0.82 & 0.81 & 0.57 & 1.00 & $|0.36|$ & 0.73 & 0.68 & 0.56 & 0.89 & 0.77 & FtHSF23 & \\
\hline 0.5 & 0.88 & 0.58 & 0.88 & 0.48 & 0.65 & 0.67 & 0.66 & \begin{tabular}{|l|l|}
0.58 \\
\end{tabular} & 0.79 & 0.94 & 0.65 & 0.84 & \begin{tabular}{|c|}
0.98 \\
\end{tabular} & 0.36 & -0.22 & 0.72 & $|-0.97|$ & $-0.94 \mid$ & 0.32 & 0.00 & $|-0.53|$ & 0.36 & 1.00 & $0.64 \mid$ & 0.07 & 0.90 & 0.74 & 0.58 & FtHSF24 & \\
\hline 0.9 & 0.63 & 0.65 & 0.51 & -0.08 & 0.05 & 0.77 & 0.56 & 0.08 & 0.57 & 0.55 & 0.50 & 0.14 & 0.70 & 0.21 & -0.48 & 0.58 & $-0.65 \mid$ & $-0.71 \mid$ & 0.44 & 0.25 & 0.16 & 0.73 & 0.64 & 1.00 & 0.05 & 0.52 & 0.80 & 0.40 & FtHSF25 & \\
\hline 0.4 & 0.14 & 0.63 & -0.06 & 0.50 & 0.58 & 0.48 & 0.75 & 0.38 & 0.33 & 0.24 & 0.71 & -0.04 & 0.22 & 0.87 & 0.66 & 0.66 & 0.15 & 0.19 & 0.87 & 0.78 & 0.49 & 0.68 & 0.07 & 0.05 & 1.00 & 0.48 & 0.55 & 0.78 & FtHSF26 & \\
\hline 0.6 & 0.86 & 0.71 & 0.70 & 0.62 & 0.83 & 0.71 & $\mid 0.87$ & 0.64 & 0.76 & 0.90 & 0.89 & 0.77 & 0.94 & $0.64 \mid$ & 0.08 & 0.92 & $-0.77 \mid$ & $-0.70 \mid$ & 0.59 & 0.36 & -0.31 & 0.56 & 0.90 & 0.52 & 0.48 & 1.00 & 0.86 & 0.87 & FtHSF27 & \\
\hline 0.8 & 0.80 & 0.82 & 0.50 & 0.29 & 0.51 & 0.84 & 0.90 & 0.34 & 0.68 & 0.71 & 0.91 & 0.37 & 0.84 & 0.62 & -0.09 & 0.94 & -0.61 & -0.60 & 0.75 & 0.61 & 0.14 & 0.89 & 0.74 & 0.80 & 0.55 & 0.86 & 1.00 & 0.86 & FtHSF28 & \\
\hline & 0.73 & 0.65 & 0.27 & 0.42 & 0.68 & 0.58 & 0.86 & 0.37 & 0.48 & 0.58 & 0.99 & 0.44 & 0.68 & 0.70 & 0.18 & 0.97 & -0.38 & -0.30 & 0.72 & $\mid 0.77$ & 0.07 & 0.77 & 0.58 & 0.40 & 0.78 & 0.87 & 0.86 & 1.00 & FtHSF29 & \\
\hline
\end{tabular}

analysis showed that Hsf in the same subgroup had similar motif composition, and contained both monocotyledonous and dicotyledonous plants in most subgroups (Fig. 6). It was speculated that $H s f$ genes appeared in monocotyledonous and dicotyledonous plants before differentiation [50, 51]. Synteny analysis showed that 19 pairs of homologous genes between Tartary buckwheat and rice were more than those between Tartary buckwheat and Arabidopsis thaliana, sugar beet and sunflower (Fig. 7, Additional file 3). This phenomenon indicates that there is no significant difference between dicotyledonous plants and monocotyledonous plants, which further indicated that the Hsf family appeared before the differentiation of monocotyledonous plants and dicotyledonous plants $[50,51]$.

\section{Functional analysis of conserved domains of FtHsf genes in Tartary buckwheat}

$H s f$ is dependent on NLS transport into the nucleus [52]. The NLS is located at the C-terminus of the HR-A/B region and the NLS is generally arginine-rich (R) and lysine-rich (K) region [53]. NES is on the C-terminal side of $H s f$, and NES plays a role in the extranuclear transport of $H s f$ [53]. The NLS and NES maintain the balance of $H s f$ in and out of the nuclear system. All FtHsfs contained the NLS domain, but only 3 FtHsrfs (FtHsf1/12/18) had an NES domain (Fig. 2, Additional file 2). Therefore, all members of the Tartary buckwheat $F t H s f$ family can play a role in the nucleus, and some of them can also travel inside and outside of the nucleus under certain conditions. The AHA motif was one of the characteristic structures of group A 


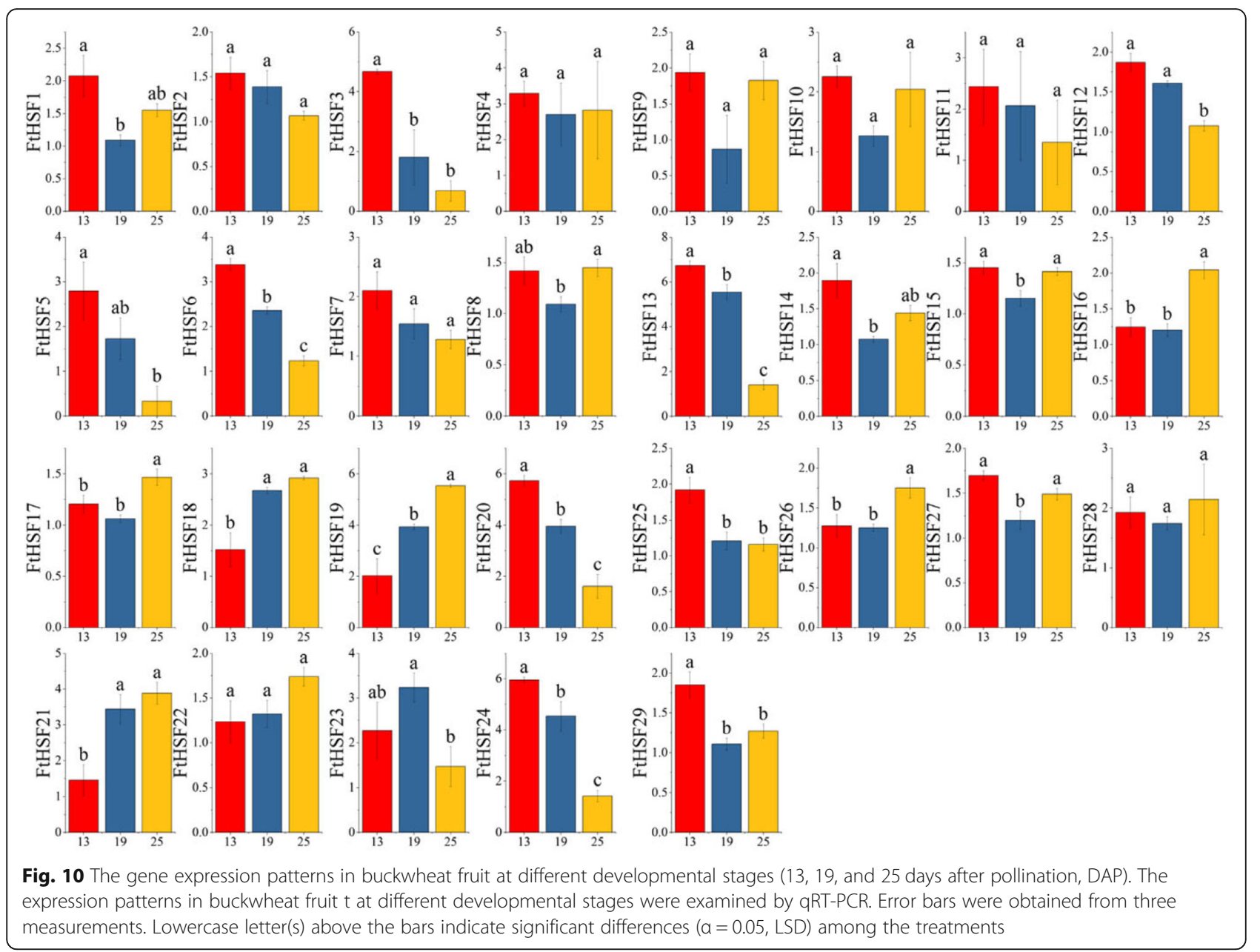

FtHsfs, and the transcriptional activation activities of group A FtHsfs were worked by the AHA of the C-terminal activation region. The AHA region is rich in aromatics, hydrophobic and acidic amino acid residues [54]. In the Tartary buckwheat Hsf family, there were 11 FtHsf members of class A with AHA motifs, and neither class B nor class $\mathrm{C}$ members contained AHA domain (Fig. 2). It was predicted that class A members with AHA domain have selftranscriptional activation activity $[55,56]$. As a result, other FtHsf members with no AHA structure didn't have transcriptional activation activities themselves, so they cannot exercise transcriptional activation alone but by forming a heteropolymer by binding to class A FtHsf to perform their functions $[55,56]$. Thus, it can be predicted that class A FtHsf members with an AHA structure play an important role in Tartary buckwheat response to an environment with high temperatures $[21,22]$.

Tartary buckwheat $\mathrm{Hsf}$ genes may play an important role in plant development

By analyzing the cis-acting elements in the promoter region of FtHsf genes, we found that the promoter region of FtHsf genes include not only Light-responsive elements and Low-temperature responsive element, but also components such as ABA-responsive elements, MeJA-responsive elements and MYB-responsive elements and so on (Fig. 2d). Which means that FtHsf genes can be involved not only in various stress responses, but also in the regulation of the growth and development of Tartary buckwheat [57]. FtHsf5 was significantly expressed in Tartary buckwheat flowers, suggesting that FtHsf5 may be involved in the development of Tartary buckwheat flowers. In a multi-species phylogenetic tree, genes in a branch usually have similar functions [50]. In Fig. 9, we found two special genes (FtHsf2O and FtHsf3) from the FtHsf genes which have a significant expression in the roots, and FtHsf2O and FtHsf3 were expressed in only three kinds of tissues. At the same time, we found that their expression showed a high positive correlation (Fig. 9). It was interesting that they belonged to the same subgroup (B4) and were closely related to the AtHsfB4 (AT1G46264.1) gene in Arabidopsis thaliana (Fig. 1). It was worth noting that the overexpression of AtHsfB4 gene in Arabidopsis 


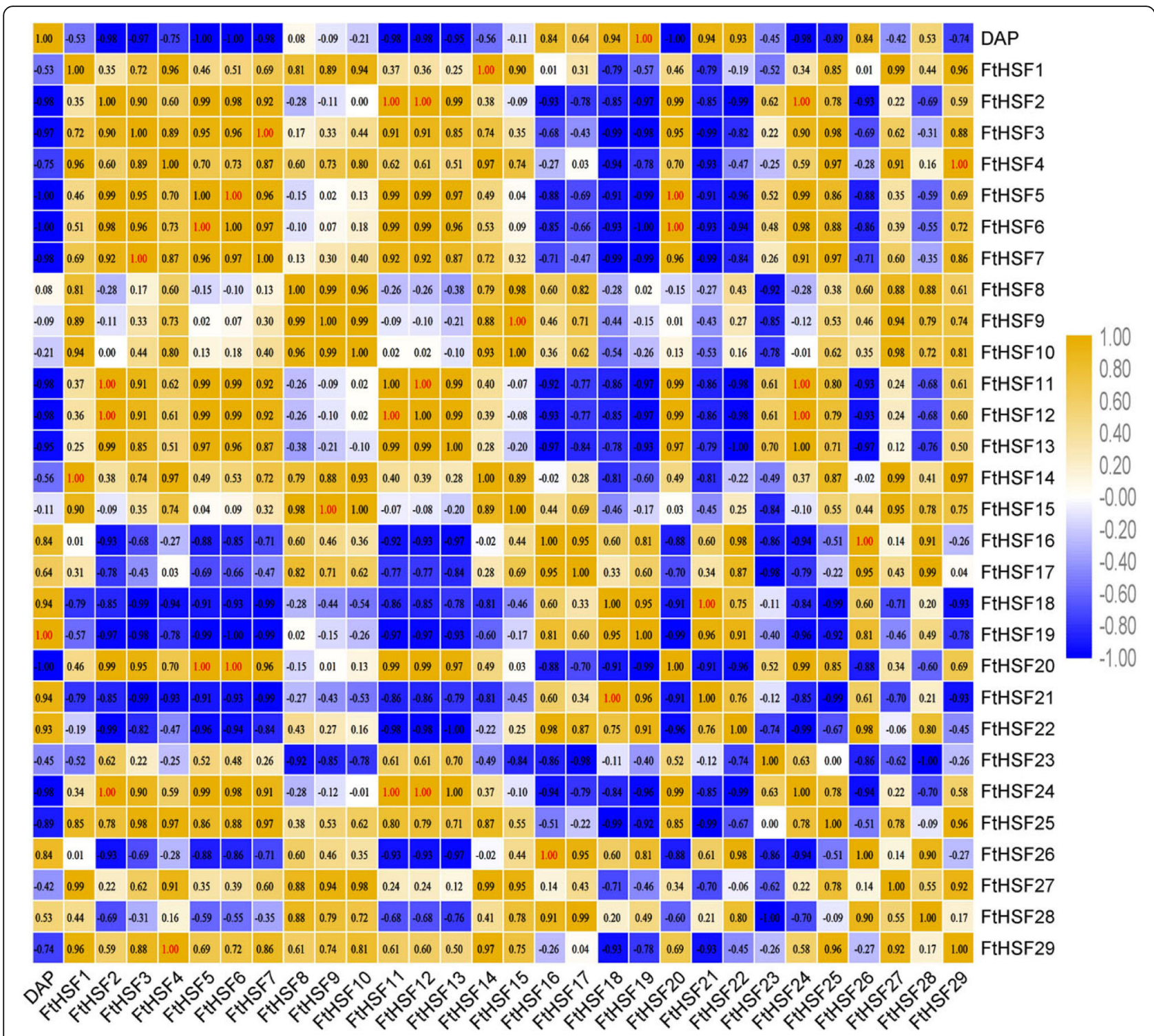

Fig. 11 The correlations between the FtHsfs genes expression patterns of buckwheat fruit at different developmental stages. Orange: positively correlated; Green: negatively correlated. The red numbers indicate a significant correlation at the 0.05 level

thaliana led to the shortening of plant root length [32], so we speculated that FtHsf 20 and FtHsf3 can also be involved in regulating the length of Tartary buckwheat root.

Meanwhile, we found that the expression levels of both FtHsf18 and FtHsf19 genes were the highest in the fruit (Fig. 8), and there was a significant positive correlation between them through Fig. 10. At the same time, by comparing the expression patterns of the FtHsf genes at different buckwheat fruit development stages (13, 19, and 25 days after pollination, DAP), founding that the expression of FtHsf18 and FtHsf19 increased gradually at the later stage of fruit development (Fig. 10). In addition, they also showed a high positive correlation in the fruit development of
Tartary buckwheat (Fig. 11). From the phylogenetic tree of Tartary buckwheat and Arabidopsis thaliana (Fig. 2), we found a close relationship between FtHsf18/FtHsf19 and AtHsfA9 (AT5G54070) in Arabidopsis. AtHsfA9 gene in Arabidopsis thaliana is regulated by the transcription factor of acid-insensitive 3 (ABI3) and participates in the regulation of fruit maturation, the higher the content of $A B A$ in fruit, the higher the expression of AtHsfA9 gene [58]. The expression patterns of AtHsfA9 in the different tissues, and at different stages of fruit development were similar to those of FtHsf 18 and FtHsf19 [5]. Meanwhile, the content of ABA during the three stages of Tartary buckwheat fruit development (13, 19, and 25 days after pollination, DAP) increased gradually, while the expression of FtHsf 18 and 


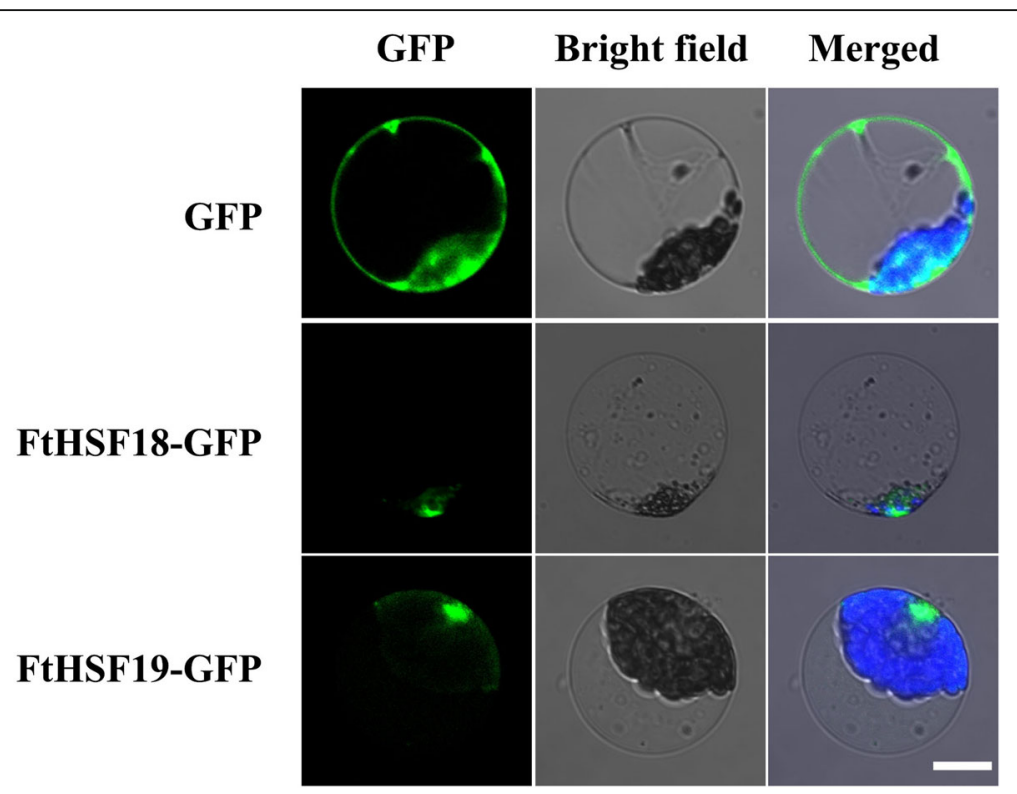

Fig. 12 Subcellular localization of FtHsf18 and FtHsf19 in Arabidopsis protoplasts. GFP and FtHsf18/19-GFP under the control of the CaMV35S promoter separately transiently expressed in Arabidopsis protoplasts

FtHsf19 during the three stages of buckwheat fruit development also increased gradually (Fig. 10) [25, 34, 58]. Therefore, we speculated that the FtHsf18 and FtHsf19genes of Tartary buckwheat may also be regulated by ABI3 and participated in the regulation of fruit ripening.

\section{Conclusions}

Overall, in this study, we found that the numbers, chromosomal locations, protein functional domains and expression patterns of 29 Tartary buckwheat FtHsf family genes are diverse and that they may be important for the plant to regulate the responses to abiotic stress and growth and development. This preliminary study on the Hsf genes of Tartary buckwheat provides a basis for further analyzing the function of the genes in different groups and for improving the stress resistance and yield of crops by using the related characteristics of the $H s f$ genes.

\section{Supplementary information}

Supplementary information accompanies this paper at https://doi.org/10. 1186/s12864-019-6205-0.

Additional file 1. List of the 29 FtHsf genes identified in this study.

Additional file 2. Analysis and distribution of conserved motifs in Tartary buckwheat and other plants Hsf proteins.

Additional file 3. One-to-one orthologous relationships between Tartary buckwheat and other seven plant species.

Additional file 4. Primers of sequences.

\section{Abbreviations}

AA: Amino acid; ABI3: Abscisic acid-insensitive3; AHA: An activator motif; DAP: Days after postanthesis; DBD: N-terminal DNA-binding domain;
GFP: Green fluorescent protein; GSDS: Gene structure display server; HMM: The hidden Markov model; HR-A/B: Composed of heptad repeats of hydrophobic amino acid residues; HSE: Heatstress element; Hsfs: Heat shock transcription factors; Hsps: Heat shock proteins; K: Lysine; LGs: Linkage groups; LSD: The least significant difference test; MADS: Minichromosome Maintenance1, Agamous, Deficiens and Serum Response Factir; Mw: Molecular weight; NBS-LRR: Nucleotide-binding site-leucine-rich repeat; NES: A nuclear export signal region; NJ: The neighbor-joining; NLS: A nuclear localization signal region; OD: An adjacent oligomerization domain; pl: Isoelectric point; qRT-PCR: Quantitative real-time polymerase chain reaction; R: Arginine; TBGP: Tartary buckwheat genome project

\section{Acknowledgements}

We thank all the colleagues in our laboratory for providing useful discussions and technical assistance. We are very grateful to the editor and reviewers for critically evaluating the manuscript and providing constructive comments for its improvement.

\section{Authors' contributions}

M-YL planned and designed the research and analyzed data. QH wrote the manuscript. QH and W-JS determined the expression of genes by qRT-PCR. Z-TM and LH identified FtHsf family genes and visualized their structures. QW and Z-ZT performed FtHsf genes chromosome distribution, gene replication and synchronous analysis. T-LB, HC and C-LL analyzed the evolutionary relationship between FtbHsf genes and several different species. HC assisted in explaining the results and revised the final version of the manuscript. $\mathrm{M}-\mathrm{YL}$ and $\mathrm{QH}$ contributed equally. All the authors have read and approved the final manuscript and ensure that it is true.

\section{Funding}

This research was supported by the National Natural Science Foundation of China (31500289), and the National Key R\&D Program of China (2018YFD1000706). Funds were used for the design of the study and collection, analysis, and interpretation of data and in writing the manuscript, as well as in the open access payment.

\section{Availability of data and materials}

The genome sequences of Tartary buckwheat used for identifying the FtHsf genes in this study were located in the Tartary Buckwheat Genome Project (TBGP; http://www.mbkbase.org/Pinku1/). The Tartary buckwheat accession (XIQIAO) materials used in the experiment were supplied by Professor Wang 
Anhu of Xichang University. All the datasets supporting the conclusions of this study are included in this article and its Additional files.

\section{Ethics approval and consent to participate}

The tartary buckwheat accession (XIQIAO) materials used in the experiment were supplied by Professor Wang Anhu of Xichang University. These plant materials are widely used all over the world and no permits are required for the collection of plant samples. The plant materials are maintained in accordance with the institutional guidelines of the College of Life Sciences, Sichuan Agricultural University, China. This article did not contain any studies with human participants or animals and did not involve any endangered or protected species.

\section{Consent for publication}

Not applicable.

\section{Competing interests}

The authors declare that they have no competing interests.

\section{Author details}

${ }^{1}$ College of Life Science, Sichuan Agricultural University, Ya'an, China. ${ }^{2}$ School of Agriculture and Biolog, Shanghai Jiao Tong University, Shanghai, China.

\section{Received: 6 January 2019 Accepted: 21 October 2019}

\section{Published online: 15 November 2019}

\section{References}

1. Wangxia W, Basia $V$, Arie A. Plant responses to drought, salinity and extreme temperatures: towards genetic engineering for stress tolerance. Planta. 2003; 218(1):1-14.

2. Mittler R. Abiotic stress, the field environment and stress combination. Trends Plant Sci. 2006;11(1):15-9.

3. Herman DJ, Knowles LO, Knowles NR. Heat stress affects carbohydrate metabolism during cold-induced sweetening of potato ( Solanum tuberosum L.). Planta. 2016;245(3):1-20.

4. Momčilović I, Pantelić D, Zdravković-Korać S, Oljača J, Rudić J, Fu J. Heatinduced accumulation of protein synthesis elongation factor $1 \mathrm{~A}$ implies an important role in heat tolerance in potato. Planta. 2016;244(3):1-9.

5. Sachin K, Elizabeth V, Helmut BU, Pascal KDR. A novel transcriptional cascade regulating expression of heat stress proteins during seed development of Arabidopsis. Plant Cell. 2007;19(1):182-95.

6. De MA. Heat shock proteins: facts, thoughts, and dreams. Shock. 1999;11(1):1-12.

7. Waters ER, Lee GJ, Vierling E. Evolution, structure and function of the small heat shock proteins in plants. J Exp Bot. 1996;47(296):325-38.

8. Mogk A, Bukau B. Role of sHsps in organizing cytosolic protein aggregation and disaggregation. Cell Stress Chaperones. 2017;22(4):1-10.

9. Santoro N, Johansson N, Dj T. Heat shock element architecture is an important determinant in the temperature and transactivation domain requirements for heat shock transcription factor. Mol Cell Biol. 1998;18(11): 6340-52.

10. Åkerfelt M, Morimoto RI, Sistonen L. Heat shock factors: integrators of cell stress, development and lifespan. Nat Rev Mol Cell Biol. 2010;11:545.

11. Yee-Yung C, Hsiang-Chin L, Nai-Yu L, Fu-Chiun H, Swee-Suak K. Arabidopsis Hsa32, a novel heat shock protein, is essential for acquired thermotolerance during long recovery after acclimation. Plant Physiol. 2006;140(4):1297-305.

12. Vierling $E$. The roles of heat shock proteins in plants. Annu Rev Plant Physiol Plant Mol Biol. 1991;42(1):579-620.

13. Sung DY, Kaplan F, Lee KJ, Guy CL. Acquired tolerance to temperature extremes. Trends Plant Sci. 2003;8(4):179-87.

14. Guo M, Liu JH, Lu JP, Zhai YF, Wang H, Gong ZH, Wang SB, Lu MH. Genome-wide analysis of the CaHsp20 gene family in pepper: comprehensive sequence and expression profile analysis under heat stress. Front Plant Sci. 2015:6(806):806.

15. Xu ZS, Li ZY, Chen Y, Chen M, Li LC, Ma YZ. Heat shock protein 90 in plants: molecular mechanisms and roles in stress responses. Int J Mol Sci. 2012; 13(12):15706-23.

16. Chapman EJ, Estelle M. Mechanism of Auxin-regulated gene expression in plants. Annu Rev Genet. 2009;43(1):265-85.

17. Bukau B, Weissman J, Horwich A. Molecular Chaperones and Protein Quality Control, vol. $125 ; 2006$
18. $\mathrm{N} \mathrm{H}, \mathrm{V}$ L. The small heat shock proteins and their clients. Cell Mol Life Sci. 2007;64(3):294-306.

19. Hartl FU, Hayer-Hartl M. Converging concepts of protein folding in vitro and in vivo. Nat Struct Mol Biol. 2009;16:574

20. Baniwal SK, Bharti K, Chan KY, Fauth M, Ganguli A, Kotak S, Mishra SK, Nover $L$, Port M, Scharf KD. Heat stress response in plants: a complex game with chaperones and more than twenty heat stress transcription factors. J Biosci. 2004;29(4):471-87.

21. Nover L, Bharti K, Döring P, Mishra SK, Ganguli A, Scharf KD. Arabidopsis and the heat stress transcription factor world: how many heat stress transcription factors do we need? Cell Stress Chaperones. 2001;6(3):177-89.

22. Li PS, Yu TF, He GH, Chen M, Zhou YB, Chai SC, Xu ZS, Ma YZ. Genomewide analysis of the Hsf family in soybean and functional identification of GmHsf-34 involvement in drought and heat stresses. BMC Genomics. 2014; 15(1):1009.

23. Lin YX, Jiang HY, Chu ZX, Tang XL, Zhu SW, Cheng BJ. Genome-wide identification, classification and analysis of heat shock transcription factor family in maize. BMC Genomics. 2011;12(1):76.

24. Fangming W, Qing D, Haiyang J, Suwen Z, Beijiu C, Yan X. Genome-wide analysis of the heat shock transcription factors in Populus trichocarpa and Medicago truncatula. Mol Biol Rep. 2012;39(2):1877-86.

25. Liu M, Ma Z, Zheng T, Wang J, Huang L, Sun W, Zhang Y, Jin W, Zhan J, Cai $Y$, et al. The potential role of Auxin and Abscisic acid balance and FtARF2 in the final size determination of Tartary buckwheat fruit. Int J Mol Sci. 2018; 19(9):2755.

26. Takanori O, Kyoko Y, Ohmi O. Two new Fagopyrum (Polygonaceae) species, F. gracilipedoides and F. jinshaense from Yunnan, China. Jpn J Genet. 2002; 77(6):399-408.

27. Anton B, Sergey N, Dmitry A, Gurevich AA, Mikhail D, Kulikov AS, Lesin VM, Nikolenko SI, Son P, Prijbelski AD. SPAdes: a new genome assembly algorithm and its applications to single-cell sequencing. J Comput Biol. 2012:19(5):455-77.

28. Danuta Z, Marcin T, Jacek K, Henryk Z. Evaluation of flavonoid contents and antioxidant capacity of the aerial parts of common and tartary buckwheat plants. Molecules. 2012;17(8):9668.

29. Schmidt R, Schippers JH, Welker A, Mieulet D, Guiderdoni E, Mueller-Roeber B. Transcription factor OsHsfC1b regulates salt tolerance and development in Oryza sativa ssp. japonica. AoB PLANTS. 2012;2012(1):pls011.

30. Jingkang G, Jian Q, Chao W, Yuan Y, Wang J, Wang Y. Genome-wide analysis of heat shock transcription factor families in rice and Arabidopsis. J Genet Genomics. 2008;35(2):105-18.

31. Scharf K-D, Berberich T, Ebersberger I, Nover $L$. The plant heat stress transcription factor ( $\mathrm{Hsf}$ ) family: structure, function and evolution. Biochim Biophys Acta. 2012;1819(2):104-19.

32. Tahmina B, Rolf R, Friedrich SF. Overexpression of AtHsfB4 induces specific effects on root development of Arabidopsis. Mech Dev. 2013;130(1):54-60

33. Wang A, Xia M, Cai G, Dai H. Buckwheat new variety Xiqiao no.2 with high yield and excellent quality. Seed. 2009;28(10):110-2.

34. Liu M, Ma Z, Wang A, Zheng T, Huang L, Sun W, Zhang Y, Jin W, Zhan J, Cai $Y$, et al. Genome-wide investigation of the Auxin response factor gene family in Tartary buckwheat (Fagopyrum tataricum). Int J Mol Sci. 2018; 19(11):3526

35. Liu M, Wen Y, Sun W, Ma Z, Huang L, Wu Q, Tang Z, Bu T, Li C, Chen H. Genome-wide identification, phylogeny, evolutionary expansion and expression analyses of bZIP transcription factor family in tartary buckwheat. BMC Genomics. 2019;20(1):483.

36. Li ZY, Xu Z-S, He G-Y, Yang G-X, Chen M, Li L-C, Ma Y-Z. A mutation in Arabidopsis BSK5 encoding a brassinosteroid-signaling kinase protein affects responses to salinity and abscisic acid. Biochem Biophys Res Commun. 2012:426(4):522-7.

37. Yoo S-D, Cho Y-H, Sheen J. Arabidopsis mesophyll protoplasts: a versatile cell system for transient gene expression analysis. Nat Protoc. 2007;2(7): 1565-72.

38. Shravan Kumar M, Joanna T, Sybille W, Bettina T, Klaus T, Lutz N, Klaus-Dieter S. In the complex family of heat stress transcription factors, HsfA1 has a unique role as master regulator of thermotolerance in tomato. Genes Dev. 2002;16(12):1555-67.

39. Mukesh K, Wolfgang B, Hannah B, Birgit K, Thorsten N, Friedrich SF. Heat shock factors $\mathrm{HsfB} 1$ and $\mathrm{HsfB} 2 \mathrm{~b}$ are involved in the regulation of Pdf1.2 expression and pathogen resistance in Arabidopsis. Mol Plant. 2009;2(1):152-65. 
40. Tatusov RL, Koonin EV, Lipman DJ. A genomic perspective on protein families. Science. 1997;278(5338):631-7.

41. Holub EB. The arms race is ancient history in Arabidopsis, the wildflower. Nat Rev Genet. 2001:2(7):516-27.

42. Zhang L, Li X, Ma B, Gao Q, Du H, Han Y, Li Y, Cao Y, Qi M, Zhu Y. The Tartary buckwheat genome provides insights into Rutin biosynthesis and abiotic stress tolerance. Mol Plant. 2017;10(9):1224-37.

43. Liu G, Chai F, Wang Y, Jiang J, Duan W, Wang Y, Wang F, Shaohua LI, Wang L. Genome-wide identification and classification of HSF family in grape, and their transcriptional analysis under heat acclimation and heat stress. Horticultural Plant J. 2018;4(04):7-17.

44. Talaee L, Fathipour Y, Talebi AA, Khajehali J. Screening of potential sources of resistance to Spodoptera exigua (Lepidoptera: Noctuidae) in 24 sugar beet genotypes. J Econ Entomol. 2016;110(1):250-8.

45. Freeling M. Bias in plant gene content following different sorts of duplication: tandem, whole-genome, segmental, or by transposition. Annu Rev Plant Biol. 2009;60(60):433-53.

46. Guo C, Guo R, Xu X, Gao M, Li X, Song J, Zheng Y, Wang X. Evolution and expression analysis of the grape (Vitis vinifera L.) WRKY gene family. J Exp Bot. 2014;65(6):1513-28.

47. Steven M, Stefanie DB, Jeroen R, Tineke C, Marc VM, Martin K, Yves VDP. Modeling gene and genome duplications in eukaryotes. Proc Natl Acad Sci U S A. 2005;102(15):5454-9.

48. Hongzhi K, Landherr LL, Frohlich MW, Jim LM, Hong M, Depamphilis CW. Patterns of gene duplication in the plant SKP1 gene family in angiosperms: evidence for multiple mechanisms of rapid gene birth. Plant J. 2010;50(5): 873-85.

49. Vision TJ, Brown DG, Tanksley SD. The origins of genomic duplications in Arabidopsis. Science. 2000;290(5499):2114-7.

50. Liu M, Ma Z, Sun W, Huang L, Wu Q, Tang Z, Bu T, Li C, Chen H. Genome-wide analysis of the NAC transcription factor family in Tartary buckwheat (Fagopyrum tataricum). BMC Genomics. 2019;20(1):113.

51. Cenci A, Guignon V, Roux N, Rouard M. Genomic analysis of NAC transcription factors in banana (Musa acuminata) and definition of NAC orthologous groups for monocots and dicots. Plant Mol Biol. 2014;85(1-2): 63-80.

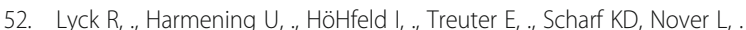
Intracellular distribution and identification of the nuclear localization signals of two plant heat-stress transcription factors. Planta 1997, 202(1):117-125.

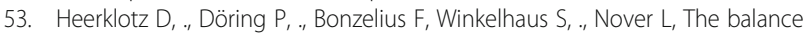
of nuclear import and export determines the intracellular distribution and function of tomato heat stress transcription factor HsfA2. Mol Cell Biol 2001, 21(5):1759-1768.

54. Cannon SB, Mitra A, Baumgarten A, Young ND, May G. The roles of segmental and tandem gene duplication in the evolution of large gene families in Arabidopsis thaliana. BMC Plant Biol. 2004;4(1):10.

55. Däöring $P$, Treuter E, Kistner $C$, Lyck R, Chen A, Nover L. The role of AHA motifs in the activator function of tomato heat stress transcription factors HsfA1 and HsfA2. Plant Cell. 2000;12(2):265-78.

56. Kotak S, Port M, Ganguli A, Bicker F, Koskull-Döring PV. Characterization of C-terminal domains of Arabidopsis heat stress transcription factors (Hsfs) and identification of a new signature combination of plant class A Hsfs with AHA and NES motifs essential for activator function and intracellular localization. Plant J. 2010;39(1):98-112.

57. Wehmeyer $\mathrm{N}$,., Vierling $\mathrm{E}_{\text {, }}$. The expression of small heat shock proteins in seeds responds to discrete developmental signals and suggests a general protective role in desiccation tolerance. Plant Physiol 2000, 122(4):1099-1108.

58. Liu M, Ma Z, Zheng T, Sun W, Zhang Y, Jin W, Zhan J, Cai Y, Tang Y, Wu Q Insights into the correlation between Physiological changes in and seed development of tartary buckwheat ( Fagopyrum tataricum Gaertn . ). BMC Genomics. 2018;19(1):648.

\section{Publisher's Note}

Springer Nature remains neutral with regard to jurisdictional claims in published maps and institutional affiliations.

Ready to submit your research? Choose BMC and benefit from:

- fast, convenient online submission

- thorough peer review by experienced researchers in your field

- rapid publication on acceptance

- support for research data, including large and complex data types

- gold Open Access which fosters wider collaboration and increased citations

- maximum visibility for your research: over $100 \mathrm{M}$ website views per year

At BMC, research is always in progress.

Learn more biomedcentral.com/submissions 